\title{
Delay or Removal of Aneurysm Formation in the Anaconda Wave Energy Extraction Device
}

\author{
Andrea Bucchi $^{(a)}$ and Grant E. Hearn ${ }^{1}$ (b) \\ (a) School of Engineering, University of Portsmouth, United Kingdom
}

(b) Fluid Structure Interaction Research Group - Faculty of Engineering and the Environment, University of Southampton, United Kingdom

\begin{abstract}
Any distensible tube inflated beyond a critical pressure will experience aneurysm formation. The Anaconda wave energy device consists of a pressurised flooded tube, which when excited with an external incident wave of appropriate frequency, for the set inflation pressure, permits generation of internal bulge waves that provide the mechanism for more efficient wave energy extraction. The distensible tube must be designed to have structural integrity and to facilitate the bulge wave matching the incident wave. The bulge wave speed is governed by internal fluid density and tube distensibility. The latter is readily shown to be dependent upon volume-pressure gradient within the tube. With application of a displacement-pressure based finite element formulation the likelihood of aneurysm and its delay or avoidance can be investigated. The strain energy functions selected for use with the finite element analysis are the Yeoh and third-order Ogden model as these formulations have been previously shown by the authors to satisfy the required Maxwell equal area rule and provide the most consistent predictions when using different mixes of experimental stress-strain data. After summarising a representative set of known wave energy extraction devices, to appreciate how different Anaconda is, the paper looks at the extent and mode of deployment of an outer inextensible reinforcement to provide bulge waves of appropriate speed whilst also overcoming the onset of aneurysms within the Anaconda tube.
\end{abstract}

Keywords: wave-energy, aneurysm avoidance, nonlinear FEA, bulge-incident wave matching

\section{Introduction}

The Anaconda wave extraction device is quite a distinctive device when compared with any other wave energy extraction devices. To appreciate how different, we identify in summary form some of the characteristics of different classes of device.

\footnotetext{
${ }^{1}$ Corresponding author, e-mail address: g.e.hearn@ soton.ac.uk School of Engineering Sciences - University of Southampton - Highfield - Southampton - SO17 1BJ - United Kingdom. +44 (0)23 80593769.
} 


\subsection{Characterising different wave energy devices}

Most wave energy devices use the relative rigid body type motions to permit energy extraction, whether they are of the 'terminator' type, such as the Salter Duck [1-4] or Lancaster Flexible bag [5] (later designated the Clam [5,6] in its ring form geometry), or assume the 'attenuator' form, such as the Cockerell Raft (sometimes referred to as Hagen-Cockerell) [7-9] or the Pelamis [10] device. That is, articulated rigid structures move relative to one another (as per Cockerell \& Pelamis devices) or like the 'ducks' or 'bags' move to react against a rigid spine. A verbal description of the Salter duck and a sketch of a 'Cockerell-device' were provided by Stahl in 1892 [8,11]. Each fluid structure interaction analysis may be undertaken by attributing rigid body like degrees of freedom to each appropriate sub-structure [9,12-17].

In the modelling of oscillating water columns [18,19], the internal free surface is usually treated as a flat rigid massless structure and it is the relative motion of the idealised free surface, relative to the floating or bottom standing structure, that is used to evaluate the air flow through the topside located turbine (Wells or others [20]). The massless plate concept can be extended to analyse damaged ships with internal free surfaces due to penetrated outer hull [21].

Devices are usually designated 'point absorbers' [22,23] when they have a representative dimension that is small relative to the incident wavelengths of interest. In a Trident-like point absorber device the modelling includes both heave rigid body fluid structure interactions with appropriate solutions of the Maxwell equations to provide the motion related power take-off damping [24,25] generated as a consequence of the reactive forces set up by magnets (built into the upper parts of the heaving structure) passing through the electrical coils to induce electrical flow. In [25] avoidance of having to retune PTO damping for different incident wave frequencies is addressed through optimization over appropriate sea spectra data sets. Devices using direct energy generation [26], such as the Lysekil device, make use of the motion of a submerged positively buoyant structure reacting against a bottom mounted mooring system [27]. An enhanced efficiency in a point absorber device can be achieved using a technique known as latching control [28].

\subsection{Characterising Anaconda}

The Anaconda device is quite different to the classes of extraction devices summarised. Apart from the PTO system it does not have any rigid body substructures that move related to one another. It 
consists primarily of a long distensible rubber tube closed at one end with a power take-off unit attached to the open end of the tube. The tube is filled with sea water and by establishing a head of water at the opened end the rubber tube is inflated to an operational design pressure.

The passage of external water waves over the pressurised water filled distensible tube, located just below the free surface, with its length orthogonal to the incident progressive water waves, leads to a pressure differential along the distensible 'tube' and the generation of internal bulge waves. The bulge waves travel just ahead of the external waves. The passage of the bulge (pressure) wave along the tube propagates energy transport within the tube. The underpinning principle of wave power extraction by Anaconda is provided by the inventors Farley \& Rainey in [29]. In order to extract a significant amount of power, $1 \mathrm{MW}$ say, the geometric dimensions of the distensible tube are likely to correspond to a length $l_{0}$ of $150 \mathrm{~m}$ and a diameter $D_{0}$ of $7 \mathrm{~m} \mathrm{[30].}$

Efficient operation of the Anaconda device requires the rubber tube to be pressurised so that the speed of the bulge wave, $c_{b}$, matches the speed of the external progressing water waves, $c_{w}$. Facilitating the matching of these two speeds requires the tube to exhibit appropriate stiffness characteristics; if the tube is too soft the bulge wave speed would be too low and if the tube is too stiff the bulge wave speed will exceed the feasible external incident wave speed. Hence resonance would not occur. Therefore achieving beneficial operational conditions requires construction of a tube with appropriate stiffness for the selected pressurisations of the tube.

\subsection{Anaconda design challenges}

Prior to the device being subjected to waves it is necessarily to ensure that the geometric and material properties of the tube and the selected induced gauge pressure (relative to the surrounding still water) are appropriate. By 'appropriate' we mean the selected internal pressure is not close to the critical pressure of the tube, so that any further increase in pressure may induce aneurysm inception. Within the literature one finds that tubes of a single material can quite readily experience aneurysms. In medical circles, where aneurysm exist due to local thinning of the 'walls', nonbiological materials must be attach at such wall locations to prevent fatalities (e.g. abdominal aortic aneurysm).

For the very large Anaconda device the investment level necessitates avoidance of the existence of aneurysm, since the resulting tube deformations could lead to dynamic behaviour inconsistent with experimental studies so far undertaken [31]. Discussions between inventors, researchers and 
industrial partners seeking to develop a prototype have concluded that only radial strain is necessary to accommodate the passage of bulge waves. Hence to match the bulge wave frequencies to the external wave environment the material properties may be adjusted using longitudinal stiffening of the tube at the different radial location so that aneurysms would exist at higher pressure level than the operational conditions. One may readily deduce from bulge wave theory introduced in Section 2.2 that increasing stiffness (through the Young modulus) leads to bulge waves that travel too fast to match the incident wave.

The existence of an aneurysm leads to significantly abrupt changes in tube cross sectional area. If one assumes that wave propagation through the tube can be modelled as one-dimensional wave (as assumed later in Section 2.2) then Lighthill in [Section 2.3 of 32] suggests the possibility of additional waves propagating in opposite directions at the cross section discontinuity. A simple interpretation of this situation is the possibility of reduced propagation of extractable energy. Farley et al. [33] appear to draw the same conclusion, in the sense of claiming aneurysms cause tube instability with uncontrollable bulges. Hence the principal concern of this paper will be selection of geometry and degree of stiffening so that the initial set up of the installed device is appropriate to permit suitable bulge wave generation without the possibility of aneurysm formation.

\subsection{Organization of the paper}

Having indicated the underpinning principles of the Anaconda device we next introduce: the fundamental concepts related to water wave speeds (bulge and external), capture width and power extraction, tube distensibility, tube behaviour with increasing inflation pressure, justification of selected strain-energy functions and estimation of resultant tube stresses. Following a proposed design modification for aneurysm reduction (removal) the extent of the parametric analysis is outlined. Results are then presented and discussed prior to paper conclusions and closure.

\section{Theoretical background}

When a water filled submerged distensible tube is subjected to the action of external water waves, behaviour of internal fluid and the structural behaviour of the tube need characterization. Each aspect is now considered in turn. 


\subsection{Water wave speed}

Successful operation of Anaconda necessitates external water wave speed matching the internal bulge wave speed.

The bulge wave speed, $c_{b}$, is controlled by the Young modulus, $E$, of the distensible material, the tube diameter, $D_{0}$, the tube wall thickness, $t_{w 0}$, and the density of the internal fluid, $\rho$.

Mathematically [34] the required relationship is: $c_{b}=\sqrt{E t_{w 0} /\left(\rho D_{0}\right)}$.

Assuming the sea waves are represented by first-order Stokes waves (Airy waves) [Section 3.2 of 35 ] then the wave frequency, $\omega$, and wavelength, $\lambda$, in water of depth $d$ must satisfy the dispersion equation $\omega^{2}=g k_{w} \tanh \left(k_{w} d\right) \quad$ : wave number $k_{w}=2 \pi / \lambda$.

The wave speed is determined from $c_{w}=\frac{\lambda}{T}=\frac{2 \pi \lambda}{2 \pi T}=\frac{\omega}{k_{w}}=\sqrt{\frac{g \tanh \left(k_{w} d\right)}{k_{w}}}$.

For deep water $\tanh \left(k_{w} d\right) \rightarrow 1$ and $c_{w}=\sqrt{g / k_{w}}$. Table 1 provides wave speeds as a function of incident wave wavelength, selected to represent fractional multiples of Anaconda tube length.

Table 1 Period and wave speed as function of wavelength

\begin{tabular}{rrr}
\hline$\lambda[\mathrm{m}]$ & $T[s]$ & $c_{w}[\mathrm{~m} / \mathrm{s}]$ \\
\hline 18.75 & 3.47 & 5.41 \\
37.50 & 4.90 & 7.65 \\
75.00 & 6.93 & 10.82 \\
100.00 & 8.00 & 12.50 \\
150.00 & 9.80 & 15.30 \\
225.00 & 12.00 & 18.74 \\
250.00 & 12.65 & 19.76 \\
300.00 & 13.86 & 21.64 \\
\hline
\end{tabular}

\subsection{Estimation of capture width and power extraction}

The arguments presented here result from reading appropriate section of Lighthill [32] and reworking analysis of Farley et al. [33]. Whilst the authors have independently solved the associated 
partial differential equation these details are not repeated here and therefore only those expressions of interest to engineering calculations are provided.

Assuming linearised water waves $u_{b}=u_{b_{-} \max } \cos \left(\omega t-k_{b} x\right)$ and hence linearised pressure, with $p_{b}=p_{b_{-} \max } \cos \left(\omega t-k_{b} x\right)$

the mean maximum bulge wave power extracted can be shown to satisfy:

$\bar{P}_{b}=\frac{1}{2} S_{o} u_{b_{-} \max } p_{b_{-} \max }$.

Here $S_{o}$ is the nominal cross sectional area of the undeformed tube. Assuming bulge speed $\left(c_{b}\right)$

matches external wave speed $\left(c_{w}\right)$ then it may be demonstrated that $u_{b_{-} \max }=p_{b_{-} \max } /\left(\rho c_{b}\right)$.

The bulge wave amplitude $B=A F$, where $A$ is the incident wave amplitude and the magnification factor $F$ at any location $x$ along the tube can be shown to satisfy: $F=\frac{1}{2} k_{w} x \equiv \pi x / \lambda$, using a simple one-dimensional wave theory.

Given both incident and bulge waves propagate in the positive x-direction we will require $\left.F\right|_{x=l_{o}}$, where $x=l_{o}$ is the power take-off end of the tube. Combining Equations (4) to (6) and noting $p_{b_{-} \max }=\rho g B$, it follows that: $\bar{P}_{b}=\frac{1}{2} S_{o} \frac{\rho g^{2} A^{2}}{c_{b}}\left(\frac{\pi x}{\lambda}\right)^{2}$.

The power available per metre width of incident wave, $P_{w}$, is given by: $P_{w}=\frac{\rho g^{2} A^{2}}{4 \omega} \equiv \frac{\rho g^{2} A^{2}}{4 k_{w} c_{w}}$.

The capture width, $C W$, of a wave energy extraction device is simply defined as the ratio of the power extracted, $\bar{P}_{b}$, and the power available per metre width of incident wave, $P_{w}$, that is:

$C W=\frac{\bar{P}_{b}}{P_{w}}=2 S_{o} k_{w}\left(\frac{c_{w}}{c_{b}}\right)\left(\frac{\pi x}{\lambda}\right)^{2}$.

Whilst Equation (9a) displays a dependence on the ratio $c_{w} / c_{b}$ most engineering calculations will treat this ratio as unity, because this corresponds to maximising energy harvesting. Hence, assuming $c_{w} / c_{b}=1$ and $\lambda=l_{o}$, then $C W$ may be expressed as: $C W=4 S_{o} \pi^{3} / \lambda \equiv \pi^{4} D_{o}^{2} / \lambda$.

To appreciate the $1 \mathrm{MW}$ power claim of Chaplin et al. [30] cited earlier we use a length $l_{0}=150 \mathrm{~m}$, $D_{0}=7 \mathrm{~m}$ and $\lambda=l_{0}$ in Equation (15a) to establish that $C W=31.82 \mathrm{~m}$. This capture width indicates that the device is expected to extract the power from a width of incident wave equal to 4.55 times 
the diameter of the device. Assuming a wave period $T=9.80 \mathrm{~s}$ and an incident wave of $A=1 \mathrm{~m}$ the power extracted is determined using: $P_{\text {extracted }}=C W \frac{\rho g^{2} A^{2}}{4 \omega} \equiv C W \frac{\rho g^{2} A^{2} T}{8 \pi}=1.22 M W$ In reality the power captured will be less since the wave system will be more complex than the one dimensional theory utilised in developing the above calculations.

\subsection{Calculating tube distensibility}

Equation (1) is equally applicable to the determination of the pulse wave speed in a human artery [34]. Just as medical science found this form of the equation to be impractical for direct use, due to the explicit dependence on Young modulus; engineering applications also require this equation to be recast. The equation modification presented next is analogous to that provided by A.E. Milne for Bramwell \& Hill [34]. For a thin walled pressure vessel, subject to a uniform pressure p, with current radius and wall thickness denoted by $r_{p}$ and $t_{w p}$, equilibrium in the circumferential direction [36] requires: $\sigma_{c}=p r_{p} / t_{w p}$.

Subsequent to an increment in uniform pressure from $p$ to $p+\Delta p$, the stress is incremented according to: $\sigma_{c}+\Delta \sigma_{c}=(p+\Delta p) r_{p} / t_{w p}$ and hence $\Delta \sigma_{c}=\Delta p r_{p} / t_{w p}$.

Assuming the simple stress-strain relationship $\Delta \sigma_{c}=E \Delta \varepsilon_{c}$, Equation (11a) can be rewritten as:

$\Delta \varepsilon_{c}=\Delta p r_{p} /\left(E t_{w p}\right)$ subject to $\Delta \varepsilon_{c}=\left(r_{p}+\Delta r_{\Delta p}-r_{p}\right) / r_{p}$.

Equating both expressions for $\Delta \varepsilon_{c}$ in Equation (11b) yields:

$\frac{\Delta r_{\Delta p}}{r_{p}}=\frac{\Delta p r_{p}}{E t_{w p}}$ and hence $\Delta r_{\Delta p}=\frac{\Delta p r_{p}^{2}}{E t_{w p}}$

The increment in pressure generates a volume change $\Delta V=l_{p} 2 \pi r_{p} \Delta r_{\Delta p} \equiv V_{p} \frac{2 \Delta r_{\Delta p}}{r_{p}}$, that is:

$$
\Delta V=V_{p} \frac{\Delta p 2 r_{p}}{E t_{w p}}=V_{p} \frac{\Delta p D_{p}}{E t_{w p}} .
$$

Since tube ends are considered fully clamped in later analysis undertaken $l_{p} \equiv l_{0}$. Upon rearranging

(13) we may introduce the distensibility parameter $\beta=\frac{1}{V_{p}} \frac{\Delta V}{\Delta p}=\frac{D_{p}}{E t_{w p}}$,

that is, bulge wave speed of Equation (1) is expressible as: $c_{b}=\sqrt{1 /(\rho \beta)}$ with $\beta=\frac{1}{V_{p}} \frac{d V}{d p}$. 
When calculating $\beta_{p}$, the distensibility at pressure $p$, the derivative in Equation (15) is evaluated as: $\beta_{p}=\frac{1}{V_{p}} \frac{V_{p+\Delta p}-V_{p}}{\Delta p}$. Replacing the forward difference based derivative estimation by a central difference representation of $d V / d p$ makes no significant impact on calculation accuracy.

\subsection{Inflation pressure influence on tube behaviour}

Having related bulge speed to tube pressure through tube distensibility we now review the impact of increased pressure on tube behaviour. During the inflation of a distensible tube a critical level of pressure may be attained. At this critical pressure 'one or more bulbous expansions' associated with the attainment of a critical radius can occur. This phenomenon is known as aneurysm. Mallock [37] is credited with presenting the first paper addressing the formation of an aneurysm in a pressurised rubber tube. Many years later Kyriakides \& Chang [38,39] provided a theoretical and experimental comparison of the formation of aneurysms in cylindrical rubber tubes. Kyriakides \& Chang observed experimentally that the tube radius expanded uniformly along the tube length with increased pressure until a critical pressure was attained. Thereafter radial growth was localized at the point of aneurysm initiation. As more fluid is injected into the tube the pressure is reduced and the aneurysm spreads longitudinally until the whole tube approximates a cylindrical shape. Further injection of fluid leads to increased pressure and radial growth until material failure occurs. The axial position of the aneurysm is influenced by manufacturing imperfections (non uniform wall thickness) or geometrical imperfections (radius variations along the tube) or even non homogeneous material properties. Aneurysm propagation requires that predicted theoretical pressure variation with radial stretch parameter, $\lambda_{1}$, should be 'N-shaped', that is, exhibits growth to a distinct maximum, reduction to a minimum and growth again so that the Maxwell equal area rule for propagation pressure can be satisfied. Meeting this requirement necessitates capture of appropriate behaviour of material through the appropriate selection of the strain-energy functions addressed next.

\subsection{Strain-energy function selection}

In a theoretical study of aneurysm initiation and propagation a strain-energy function is required to describe stress-strain behaviour of the distensible tube investigated. Many different choices of strain-energy functions are available. A common choice in engineering [38-45] and in medical 
applications [46-48] is represented by an Ogden model [49]. In a general study the authors [50,51] have established that:

(i) The third-order Ogden and the Yeoh [52] strain-energy functions were preferred, since they are both capable of satisfying the Maxwell equal area requirement [43] associated with aneurysm propagation.

(ii) Combining uniaxial and equi-biaxial data or uniaxial, equi-biaxial and pure shear data sets provided the most consistent predictions of critical pressure and inflation behaviour of the distensible tube.

The preferred Ogden and Yeoh strain energy functions, $W$, have the following respective forms:

$W=\sum_{i=1}^{N} \frac{2 \mu_{i}}{\alpha_{i}^{2}}\left(\lambda_{1}^{\alpha_{i}}+\lambda_{2}^{\alpha_{i}}+\lambda_{3}^{\alpha_{i}}-3\right)$

and

$W=C_{10}\left(I_{1}-3\right)+C_{20}\left(I_{1}-3\right)^{2}+C_{30}\left(I_{1}-3\right)^{3}$.

For an isotropic incompressible material the principal stretches $\lambda_{i}: i=1,2 \& 3$, for uniaxial, equibiaxial and pure shear stretching satisfy respectively:

$\lambda_{1}=\lambda$ and $\lambda_{2}=\lambda_{3}=1 / \sqrt{\lambda}$,

$\lambda_{1}=\lambda_{2}=\lambda$ and $\lambda_{3}=1 / \lambda^{2}$,

$\lambda_{3}=1, \lambda_{1}=\lambda$ and $\lambda_{2}=1 / \lambda$,

and the first strain invariant is defined as $I_{1}=\lambda_{1}^{2}+\lambda_{2}^{2}+\lambda_{3}^{2}$.

The $N$ parameter pairs $\alpha_{i}, \mu_{i}$ and the coefficients $C_{10}, C_{20}, \& C_{30}$ are numerically fitted for each cited combination of data sets in a least-square sense [50,53]. Use of the selected strain-energy functions will permit theoretical monitoring of an inflated distensible tube. As pressure increases so the stress distribution will be modified as a consequence of radial stretch and tube thickness variation. Estimation of resultant material stress levels therefore requires our attention.

\subsection{Monitoring resultant material stresses}

In general, using Cartesian coordinates, the von Mises stress [54, article 78] is expressed as:

$2 \sigma_{v}^{2}=\left(\sigma_{x x}-\sigma_{y y}\right)^{2}+\left(\sigma_{y y}-\sigma_{z z}\right)^{2}+\left(\sigma_{x x}-\sigma_{z z}\right)^{2}+6\left(\sigma_{x y}^{2}+\sigma_{x z}^{2}+\sigma_{y z}^{2}\right)$, 
and the acceptability of this stress is achieved by ensuring that $\sigma_{v} \leq \alpha \sigma_{T}$ with $\sigma_{T}$ denoting tensile yield stress and $0<\alpha<1$, depending upon the safety factor required for the application. In terms of principal stress components Equation (19a) simplifies to:

$2 \sigma_{v}^{2}=\left(\sigma_{1}-\sigma_{2}\right)^{2}+\left(\sigma_{2}-\sigma_{3}\right)^{2}+\left(\sigma_{1}-\sigma_{3}\right)^{2}$.

A variation of this criterion for polymers has been suggested [55]. In particular, the estimated resultant stress may be determined from:

$2 \sigma_{v}^{2}=\left(\sigma_{1}-\sigma_{2}\right)^{2}+\left(\sigma_{2}-\sigma_{3}\right)^{2}+\left(\sigma_{1}-\sigma_{3}\right)^{2}+2\left(\sigma_{C}-\sigma_{T}\right)\left(\sigma_{1}+\sigma_{2}+\sigma_{3}\right)$

and in this case it is required that $\sigma_{v} \leq \alpha \sqrt{\sigma_{C} \sigma_{T}}$, where $\sigma_{C}$ is the compressive yield stress. For the experimental data sets to be used [56,57] the compressive and tensile yield stresses are not recorded. Hence the pragmatic approach of utilising Equation (19b) is to be adopted when looking at the resulting stress variation for different scenarios. This is sufficient to appreciate the relative stress changes as each different situation is monitored. In the ultimate verification of Anaconda feasibility it would be necessary to undertake a more considered and detailed experimental investigation of the selected rubber so that an effective sizing criterion can be applied. There is also a need to establish for the rubber selected that Equation (19c) is an appropriate generalization of (19b).

Having introduced the basic definition required to undertake the intended calculations we next consider how aneurysm prevention or postponement may be achieved.

\subsection{Proposed method of aneurysm delay or removal}

Aneurysm postponement or prevention requires modification of tube construction and behavioural characteristics. It is proposed that longitudinal strips of an inextensible material are added to enlarge tube stiffness whilst simultaneously permitting exploitation of the strain levels achievable with pure rubber sections to permit generation and propagation of bulge waves at the correct speed. This opening gambit requires clarification in terms of rubber selected, number of external strips used, relative coverage of tube by inextensible material, thickness of rubber tube and reinforcements with due considerations of stress-levels associated with each possible tube construction. Since the proposed tube modifications are most readily analysed using finite elements, we next consider constraints upon finite element selection and the underpinning principles of the analysis undertaken. 


\section{Finite element analysis}

Finite element methods may be readily employed to study aneurysm formation and propagation. Shi $\&$ Moita [40] developed their own axisymmetric hyper-elastic membrane and solved the non-linear static problem with the arc-length method [58]. The authors [50,51] compared their predictions with the independent Shi \& Moita study to provide confidence in the application of the selected commercial software package used. Each of these static analysis neglects hysteresis effect, since only strain stress data is utilised to address strain energy function specification in the adopted static analysis. There is no consideration of strain rate, which under very limiting conditions may be equated to pressure change rate and thereafter ignored [33]. Hence hysteresis is not explicitly addressed here.

Equations (18) assume that the rubber material considered is incompressible with the Poisson ratio at the limit value of 0.5 . This physical constraint necessitates a mixed or hybrid displacement/pressure (U/P) finite element formulation [59]. The impact of the strain-energy function selection upon the associated stiffness matrix of a U/P formulation is partially addressed in [50]. Earlier studies [51] demonstrated that more than one element through the wall thickness of a rubber tube was unnecessary. For the two-material layered tube the rubber wall thickness is represented by one solid element with an additional solid element used to model the outer attached inextensible added $\operatorname{skin}^{2}$. The particular linear solid element selected was the ABAQUS ${ }^{\circledR} \mathrm{C} 3 \mathrm{D} 8 \mathrm{H}$ for the rubber and the C3D8 for the reinforcement. Since the inflation pressure is uniform, exploiting geometric symmetry only requires modelling of one quarter of the tube. Given clamped tube end-conditions are symmetrically applied to both ends, modelling 1/8 of the tube is now sufficient. Exploitation of geometric symmetry can be shown to readily capture tube behaviour [51]. Details of analysis and element discretisation are discussed next.

\subsection{Organization of parametric analysis}

Ideally a wide variety of different rubbers would be tested to indicate both choices and sensitivity of behaviour to material composition. In reality the complete data sets available are those due to Treloar (1944) [56] and Kawabata et al. (1981) [57]. Data provided by Rivlin \& Saunders [60] is unacceptable because it relates to two rubbers of slightly different preparations; James, Green \&

\footnotetext{
${ }^{2}$ Method of securing the outer inextensible skin to the rubber, or, the alternative construction of single layers of rubber and inextensible material joints via a 'seam' require (experimental) investigation beyond the theoretical analysis of this paper.
} 
Simpsons [61] and James \& Green [62] provide limited tabulated data for two distinct rubbers. In consequence we can only consider the historical data sets of Treloar [56] and Kawabata et al. [57]. It is assumed that the reported data is consistent with standard measurement techniques, whereby stress strain data recorded corresponds after appropriate cyclic loading of material, so that hysteresis effects have been minimalized. For a totally distensible tube it has been shown [50,51] that consistent predictions may be established using the uniaxial, equi-biaxial \& pure shear data sets or the uniaxial \& equi-biaxial data sets combination. This approach is also to be used with the proposed partially stiffened distensible tube. However, since uniaxial data is the more common measurement approach, this data set is included to demonstrate the wider possible variation in predictions. Experimental measurement of pressure versus radial stretch ratio, $\lambda_{1}$, suggests an 'Nshaped' curve. Consequently only the Yeoh and third-order Ogden models are used since they fulfil this requirement and also permit estimation of propagation pressure via the Maxwell equal area rule [43].

The parametric analysis undertaken is driven by the need to avoid aneurysm initiation whilst achieving a desirable range of bulge wave velocities for optimization of energy extraction in different sea-states. To maintain symmetric deformations of the circular distensible tube, as inflation pressure is uniformly increased, the number of reinforcement strips considered is $4,8 \&$ 16. To vary the range of attainable bulge wave velocities rubber wall thickness assumes values of $7 \mathrm{~cm}, 14 \mathrm{~cm}$ and $28 \mathrm{~cm}$. Reinforcement thickness is nominally $3 \mathrm{~cm}$ with alternative thicknesses of $1 \mathrm{~cm}$ and $5 \mathrm{~cm}$ also addressed to demonstrate that rubber thickness, rather than reinforcement thickness, is the principal parameter governing the range of bulge wave velocities. The ratio $t_{w 0} / r_{0}$ is consistent with the range of values addressed earlier [51] and consistent with a single element model $\left(N_{t}=1\right)$ for the wall thickness. The Nylon-like material [63] representing the reinforcement is considered as an isotropic material with a Young modulus of $3 \mathrm{GPa}$ and a Poisson ratio of 0.35 . The Young modulus of the reinforcement material is roughly three orders of magnitude larger than the 'Young modulus' of the rubber material. However, variation of the reinforcement Young modulus is varied to demonstrate it has negligible impact on the overall behaviour of the reinforced distensible tube. Having indicated the basis of the parametric analysis to be reported, we now present details of the finite element discretisation of the explicitly modelled portion of the tube.

Each finite element models an arc length corresponding to an angle of $4.5^{\circ}$, that is, there are $N_{c}=20$ elements per quadrant circumferentially. For a half tube length of $75 \mathrm{~m}$ the longitudinal 
discretisation of the tube uses $N_{l}=272$ elements to create an element aspect ratio approaching unity. For 4 reinforcement strips the total angle covered by the inextensible material is incremented in steps of $36^{\circ}$ over the range $36^{\circ}$ to $324^{\circ}$. The corresponding number of solid elements to model the reinforcement circumferentially increases from $N_{c}^{R}=2$ to 18 in steps of 2 per quadrant. So the number of pure rubber elements is $N_{c} \times N_{l} \times N_{t}=5440$ when modelling just $1 / 8$ of the structure. The number of reinforcement elements varies from $N_{c}^{R} \times N_{l}=544$ in increments of 544 up to 4896 for $N_{c}^{R}=18$. Division of circumference into different numbers of reinforcement strips is illustrated in Figure 1 together with an indication of a corresponding finite element discretisation for 8 reinforcement strips. With the intended extent of the investigation explained, results of the parametric studies are presented and discussed next.

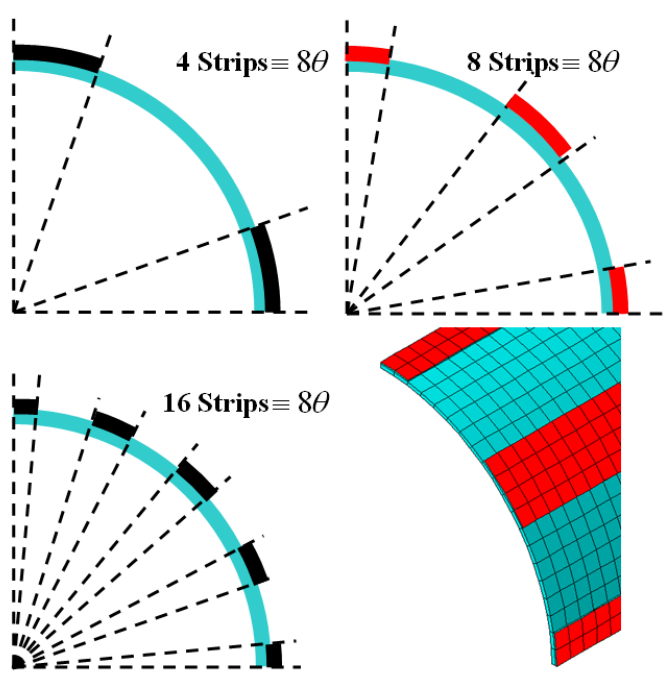

Fig. 1 Conceptual and FE model of reinforced distensible tube

\section{Presentation and discussion of predictions}

This section will address in turn: aneurysm avoidance, device tuning, influence of reinforcement characteristics and structural integrity.

\subsection{Avoidance of aneurysm inception}

Figure 2 indicates the sensitivity of the inflation pressure as a function of radial stretch parameter $\lambda_{1}$ for the Treloar (a, c \& e) and the Kawabata et al. (b, d \& f) based rubbers for different total coverage angles of reinforcement material divided into 4 symmetrically spaced strips. The earlier 
selected rubber wall thicknesses of $7 \mathrm{~cm}, 14 \mathrm{~cm}$ and $28 \mathrm{~cm}$, with reinforcement thickness of $3 \mathrm{~cm}$, are analysed using the third-order Ogden and the Yeoh strain-energy functions of Equations (16 \& 17). For comparison the behaviour of a pure rubber tube $\left(0^{\circ}\right)$ is included in each plot. Figures $2 \mathrm{e} \& \mathrm{f}$ have been reconstituted as Figures $2 \mathrm{~g} \& \mathrm{~h}$ so that variations in rubber for a given strain-energy function can be readily appreciated.

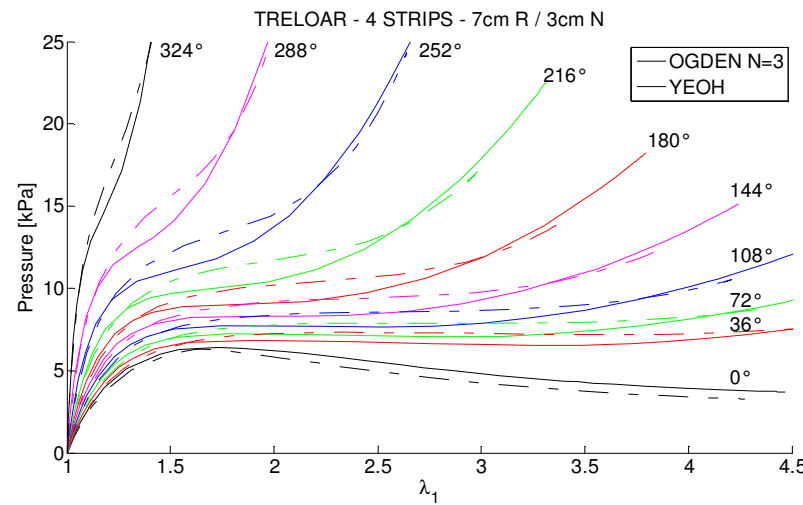

Fig. 2a Influence of reinforced material coverage on Treloar rubber of thickness $7 \mathrm{~cm}$

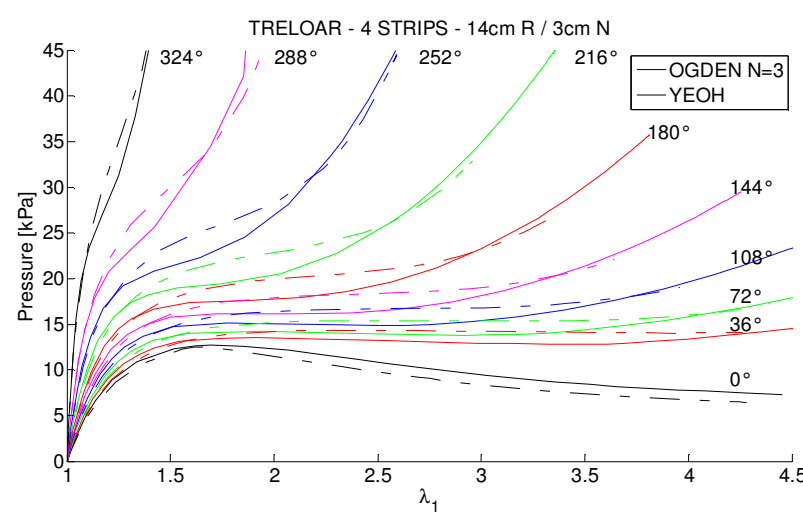

Fig. 2c Influence of reinforced material coverage on Treloar rubber of thickness $14 \mathrm{~cm}$

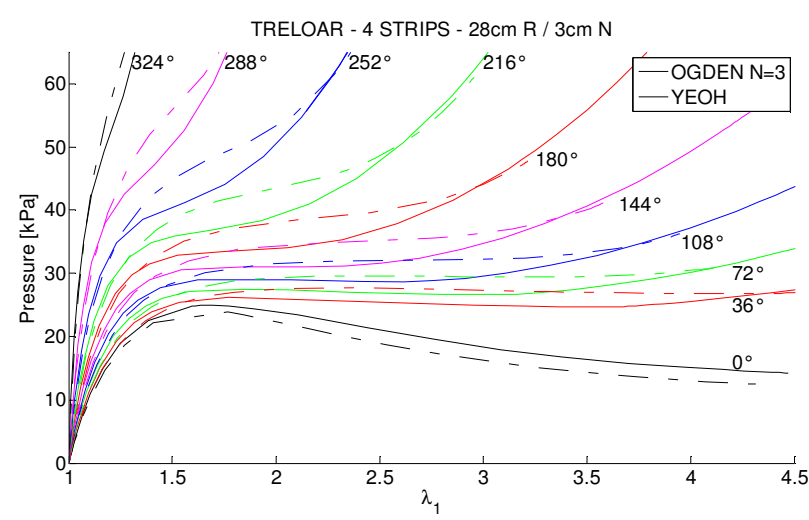

Fig. 2e Influence of reinforced material coverage on Treloar rubber of thickness $28 \mathrm{~cm}$

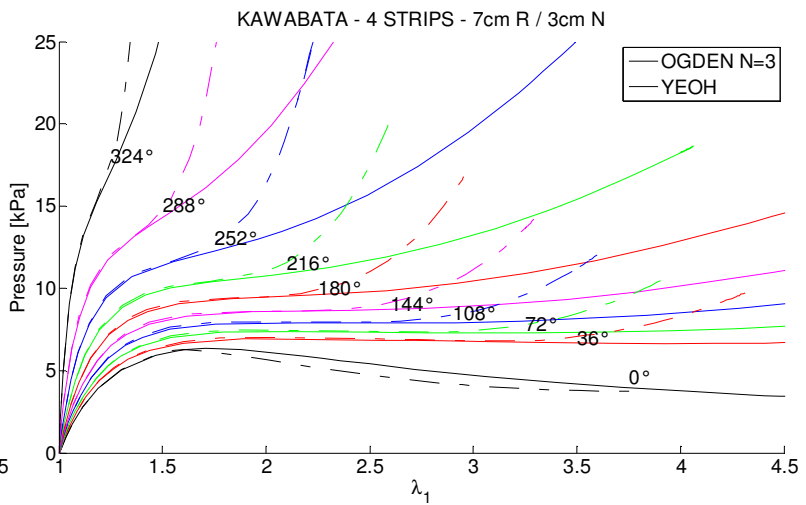

Fig. 2b Influence of reinforced material coverage on Kawabata rubber of thickness $7 \mathrm{~cm}$

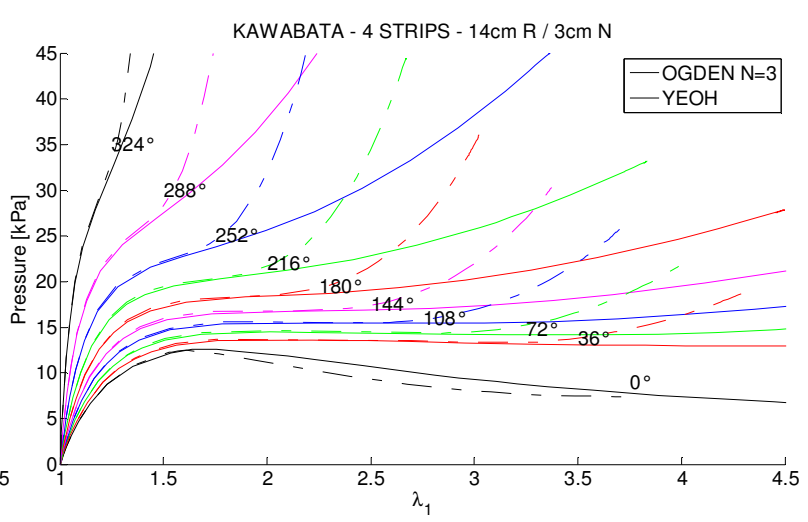

Fig. 2d Influence of reinforced material coverage on Kawabata rubber of thickness $14 \mathrm{~cm}$

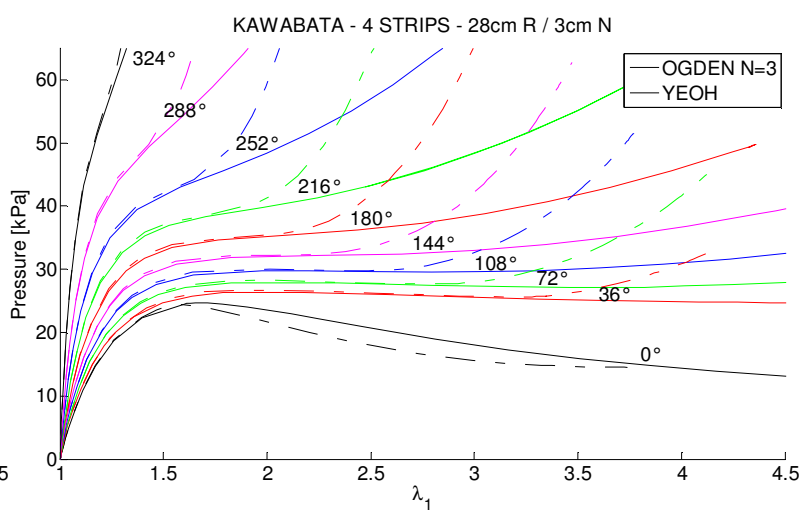

Fig. $2 f$ Influence of reinforced material coverage on Kawabata rubber of thickness $28 \mathrm{~cm}$ 


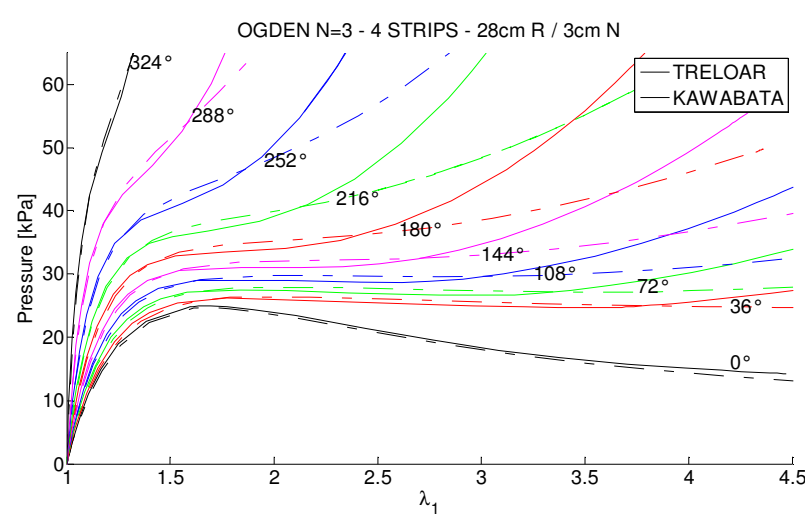

Fig. 2g Influence of reinforced material coverage on Treloar \& Kawabata rubbers of $28 \mathrm{~cm}$ thickness for Ogden ( $N=3$ ) strain-energy model

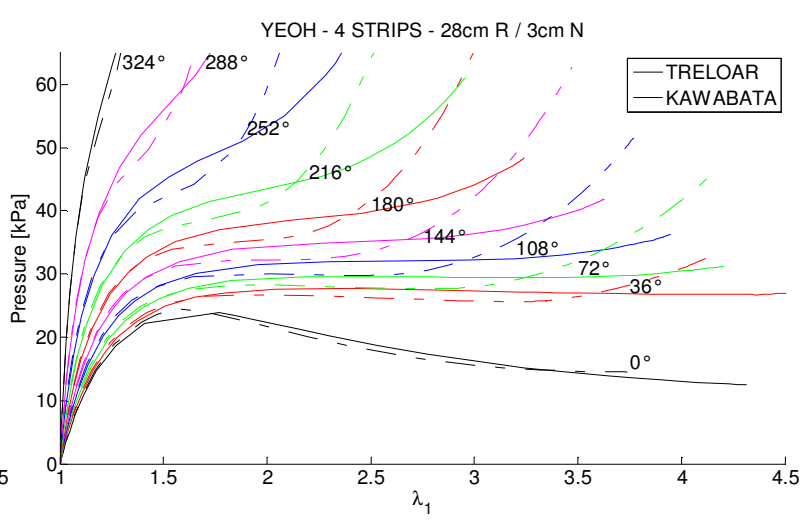

Fig. $2 \mathrm{~h}$ Influence of reinforced material coverage on Treloar \& Kawabata rubbers of $28 \mathrm{~cm}$ thickness for Yeoh strain-energy model

Figure 2 clearly shows that as the total coverage angle increases so the likelihood of aneurysm initiation is significantly delayed or removed. In fact, for a coverage angle less than $144^{\circ}$ a critical pressure may exist. Furthermore, as the percentage of tube coverage with reinforcement material increases, the existence of a peak critical inflation pressure is transformed into a 'plateau' pressure, which in turn becomes a monotonically increasing inflation pressure. For the stiffened Treloar rubber, the influence of a selected and fitted strain-energy function upon the resulting behaviour is significantly less sensitive than the corresponding variation associated with the stiffened Kawabata et al. rubber. For each total coverage angle the Kawabata rubber exhibits a clearly defined 'bifurcation' point regarding $p-\lambda_{1}$ variations.

We now investigate whether this observation is affected when varying the number of reinforcement strips. The $4.5^{\circ}$ arc coverage of each finite element restricts investigation to comparison of $4,8 \&$ 16 strips of reinforcement material and total coverage angles of $144^{\circ}$ or $288^{\circ}$. Figure 3 illustrates the variation of pressure with radial stretch parameter for both rubbers and the preferred strainenergy functions. 


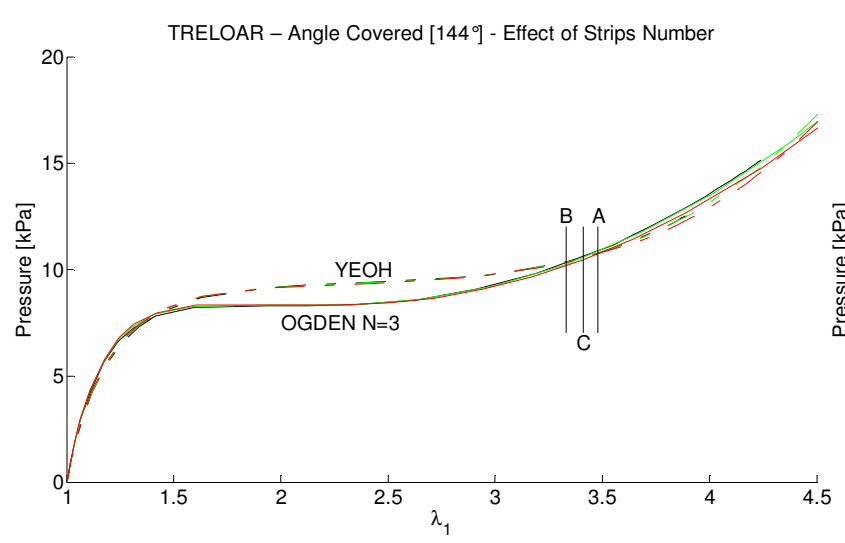

Fig. 3a $p-\lambda_{1}$ for $4,8 \& 16$ strips with reinforcement coverage of $144^{\circ}$ for Treloar rubber

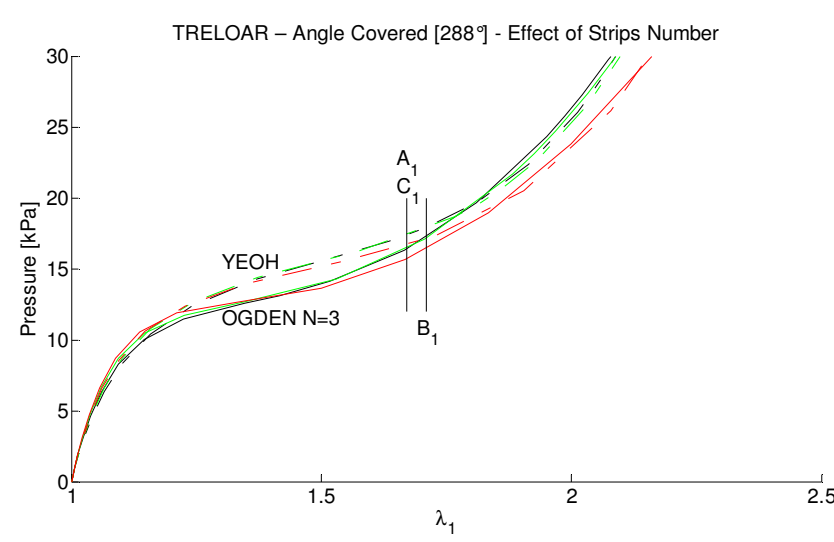

Fig. 3c $p-\lambda_{1}$ for $4,8 \& 16$ strips with reinforcement coverage of $288^{\circ}$ for Treloar rubber

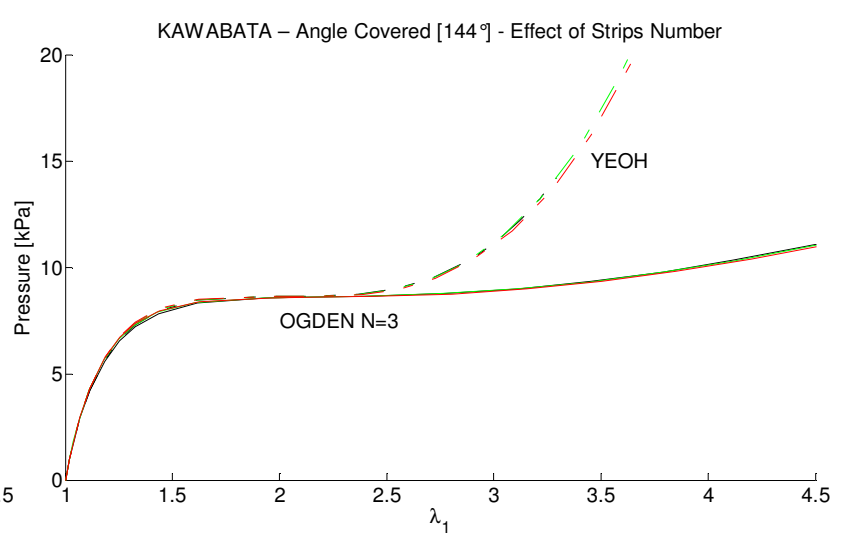

Fig. 3b $p-\lambda_{1}$ for $4,8 \& 16$ strips with reinforcement coverage of $144^{\circ}$ for Kawabata rubber

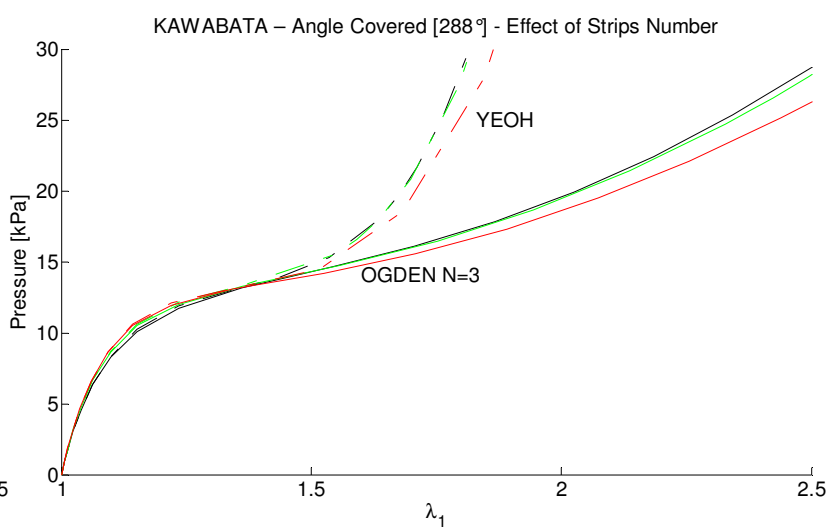

Fig. 3d $p-\lambda_{1}$ for $4,8 \& 16$ strips with reinforcement coverage of $288^{\circ}$ for Kawabata rubber

Figures $3 \mathrm{a} \& \mathrm{~b}$ for a total coverage angle of $144^{\circ}$ indicate that differences do arise as a consequence of fitting the different strain-energy functions, but for a selected strain-energy function the influence of the number of strips used (as defined in Figure 1) is apparently insignificant. The same conclusions can be drawn regarding the total coverage angle of $288^{\circ}$ in Figures $3 \mathrm{c} \&$ d. Hence, 4 strips may be selected as the simplest method of maintaining symmetric expansion of the tube. Comparison of Figure $3 a$ with Figure $3 \mathrm{c}$ and comparison of Figure $3 \mathrm{~b}$ with Figure $3 \mathrm{~d}$ indicate that the pressure slope significantly increases as the total coverage angle becomes larger.

\subsection{Tuning device to achieve appropriate bulge wave speed}

In this parametric analysis the authors will illustrate, for the first time, how the gauge pressure can be used to achieve matching of bulge wave speed to incident wave speed to enhance energy extraction efficiency. 


\subsubsection{Bulge wave speed dependency on wall thickness and coverage angle}

Using 4 strips of reinforcement the pressure-volume curves, necessary to estimate the distensibility $\beta$ of Equation (15), are presented together with estimates of bulge wave speed $c_{b}$ versus pressure for the Treloar rubber with wall thicknesses of $7 \mathrm{~cm}, 14 \mathrm{~cm}$ and $28 \mathrm{~cm}$ and a fixed reinforcement thickness of $3 \mathrm{~cm}$. Again the strain-energy function and the total reinforcement coverage angle are varied. The described parametric study provides Figures $4 \mathrm{a}$ to $\mathrm{f}$. Figure $4 \mathrm{f}$ also includes the additional coverage angle of $324^{\circ}$, whereas Figures $4 \mathrm{~g} \& \mathrm{~h}$ provide reworked solutions for a wall thickness of $28 \mathrm{~cm}$ using the Kawabata et al. rubber.

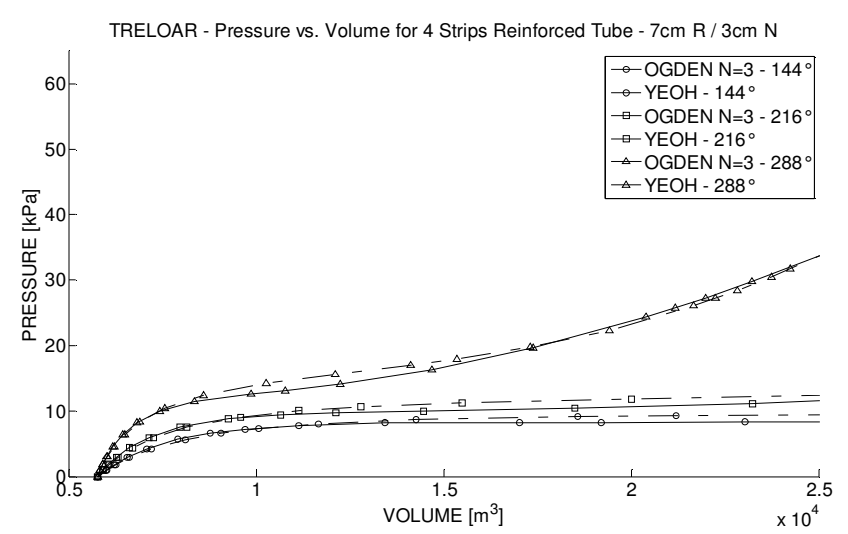

Fig. 4a P-V variation for $144^{\circ}, 216^{\circ} \& 288^{\circ}$ for $7 \mathrm{~cm}$ thick Treloar rubber

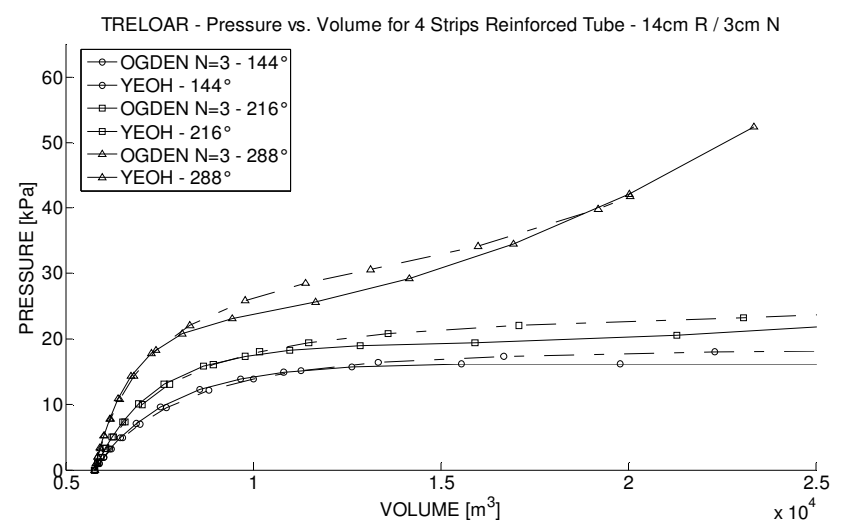

Fig. 4c P-V variation for $144^{\circ}, 216^{\circ} \& 288^{\circ}$ for $14 \mathrm{~cm}$ thick Treloar rubber

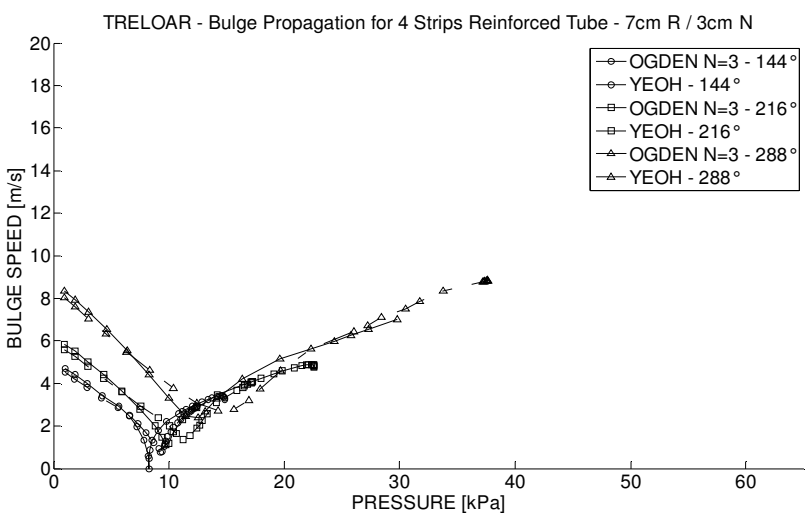

Fig. 4b Bulge speed variation for $144^{\circ}, 216^{\circ} \& 288^{\circ}$ for $7 \mathrm{~cm}$ thick Treloar rubber

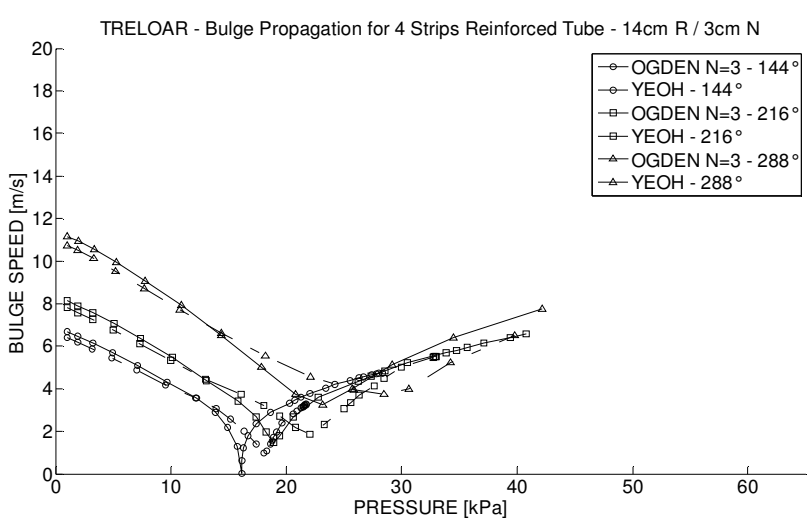

Fig. 4d Bulge speed variation for $144^{\circ}, 216^{\circ} \& 288^{\circ}$ for $14 \mathrm{~cm}$ thick Treloar rubber 


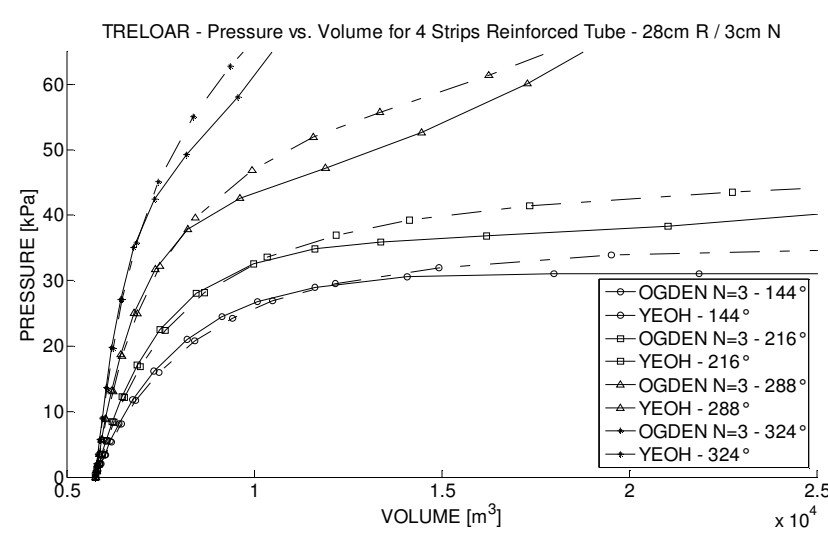

Fig. 4e P-V variation for $144^{\circ}, 216^{\circ}, 288^{\circ} \& 324^{\circ}$

for $28 \mathrm{~cm}$ thick Treloar rubber

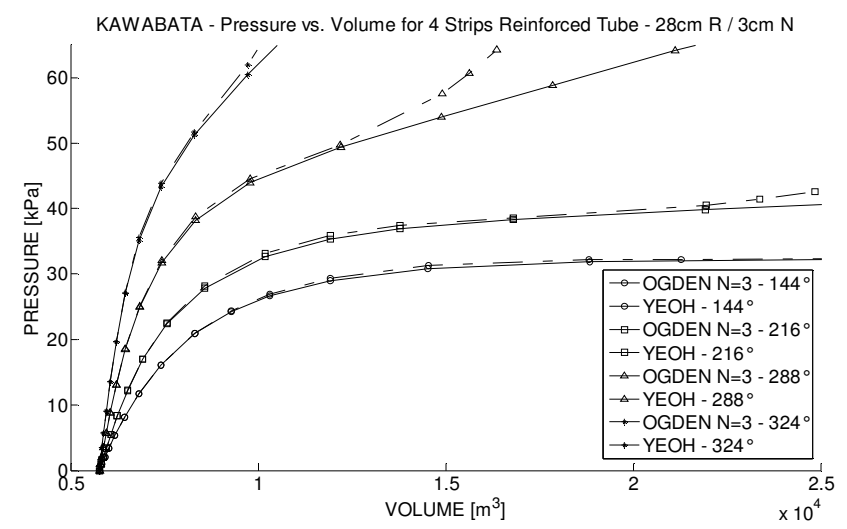

Fig. 4g P-V variation for $144^{\circ}, 216^{\circ}, 288^{\circ} \& 324^{\circ}$

for $28 \mathrm{~cm}$ thick Kawabata rubber

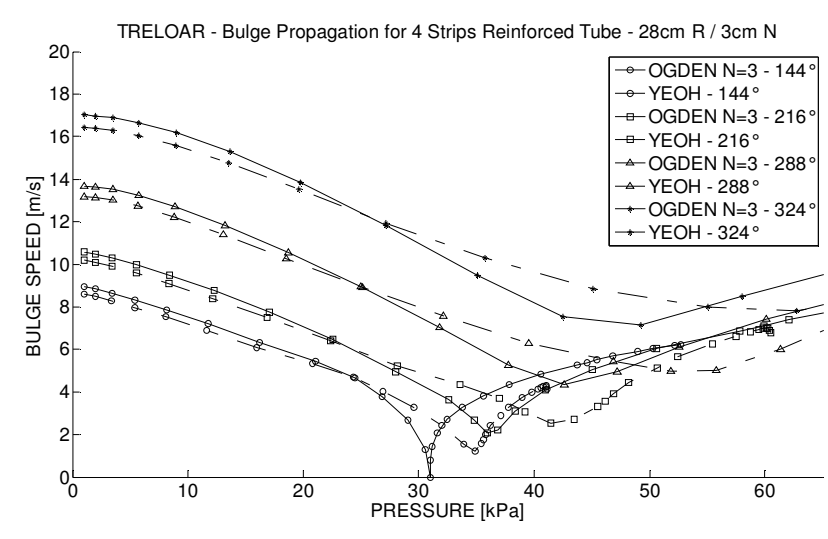

Fig. 4f Bulge speed variation for $144^{\circ}, 216^{\circ}, 288^{\circ} \&$

$324^{\circ}$ for $28 \mathrm{~cm}$ thick Treloar rubber

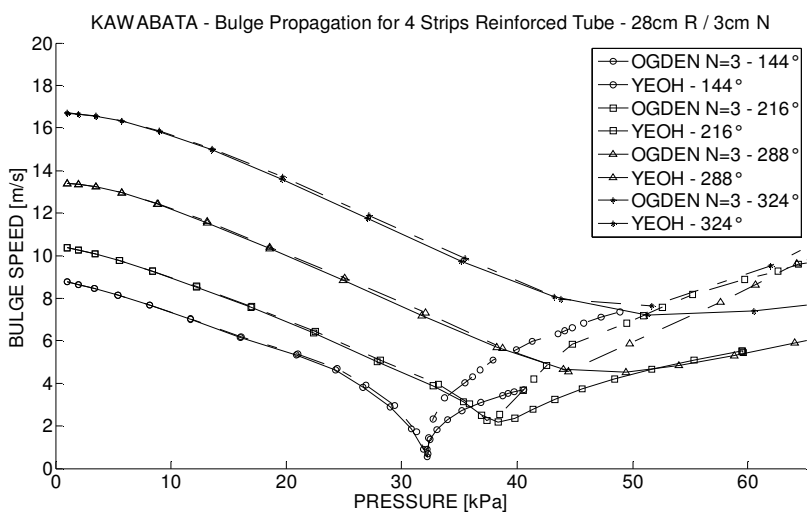

Fig. 4h Bulge speed variation for $144^{\circ}, 216^{\circ}, 288^{\circ} \&$ $324^{\circ}$ for $28 \mathrm{~cm}$ thick Kawabata rubber

Figures $4 \mathrm{a}, \mathrm{c} \& \mathrm{e}$ indicate that the pressure associated with a given volume increases with material thickness and coverage angle of the reinforcement material. These two geometric variables are responsible for the observable differences between the Yeoh and Ogden models as pressure is increased. However, Figures $4 \mathrm{~b}, \mathrm{~d} \& \mathrm{f}$ suggest that the differences in bulge wave speed due to different strain-energy functions selected are generally small compared to differences due to different rubber thicknesses. Figures $4 \mathrm{~g} \& \mathrm{~h}$ should be directly compared with Figures $4 \mathrm{e} \& \mathrm{f}$. The former pair of figures indicates that $p-V$ variation and $c_{b}-p$ variation for the Kawabata material are significantly less sensitivity to strain-energy function choice than the corresponding Treloar material results of Figure $4 \mathrm{e} \& \mathrm{f}$. This observation is consistent with the findings presented in [51]. Figures $4 b, d \&$ f clearly indicate that to increase bulge wave speed it is necessary to increase: (i) rubber thickness or (ii) angle of reinforcement coverage or (iii) both. For a given thickness one notices that bulge wave speed changes associated with increasing reinforcement coverage from $216^{\circ}$ to $288^{\circ}$ are twice the speed changes associated when reinforcement coverage increases from $144^{\circ}$ to $216^{\circ}$. Furthermore, for a given reinforcement coverage angle, the bulge wave speed changes 
with the square root of rubber thickness as suggested by Equation (1). Figure $4 \mathrm{f}$ indicates that we attain the same level of increase in bulge wave speed by increasing coverage from $288^{\circ}$ to $324^{\circ}$ (i.e. $36^{\circ}$ ) as that achieved when increasing coverage between $216^{\circ}$ and $288^{\circ}$ (i.e. $72^{\circ}$ ). The von Mises stresses of Figures 8 to 11 (to be presented) should help us to decide how increases in thickness and reinforcement coverage should be managed to achieve preferred bulge wave speeds.

\subsubsection{Impact of rubber characteristics on bulge wave speed}

In all the results presented the strain-energy functions selected have been fitted using uniaxial (U), equi-biaxial (E) and pure shear data (P). Removal of the pure shear data has little effect upon a pure rubber tube [51]. Uniaxial data corresponds to the simplest measurement technique and is considered sufficient by many practitioners.

In Figure 5 the influence of selected data sets of Treloar (5a) and Kawabata (5b) rubbers used to fit the strain-energy function parameters is investigated for both Yeoh and third-order Ogden models. In each case rubber wall thickness is $28 \mathrm{~cm}$, there is $3 \mathrm{~cm}$ of reinforcement and the coverage angle is $288^{\circ}$.

The application of the Ogden model for both the Treloar and Kawabata materials provide consistent variation of bulge wave speed with pressure (up to $40 \mathrm{kPa}$ ) whether using the U/E/P or U/E combinations of experimental data. For the Yeoh model and the Treloar material consistency begins to wane beyond $30 \mathrm{kPa}$, whereas for the Kawabata data real differences do not exist for pressure less than $45 \mathrm{kPa}$. Beyond this last threshold variation with different data sets is more significant for the Kawabata material than for the Treloar material.

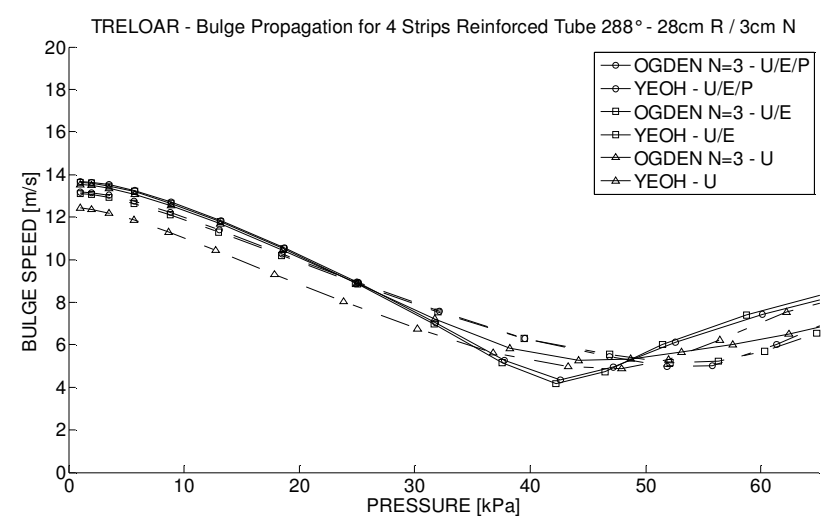

Fig. 5a Bulge wave speed sensitivity of Treloar rubber to selected experimental data sets used to fit

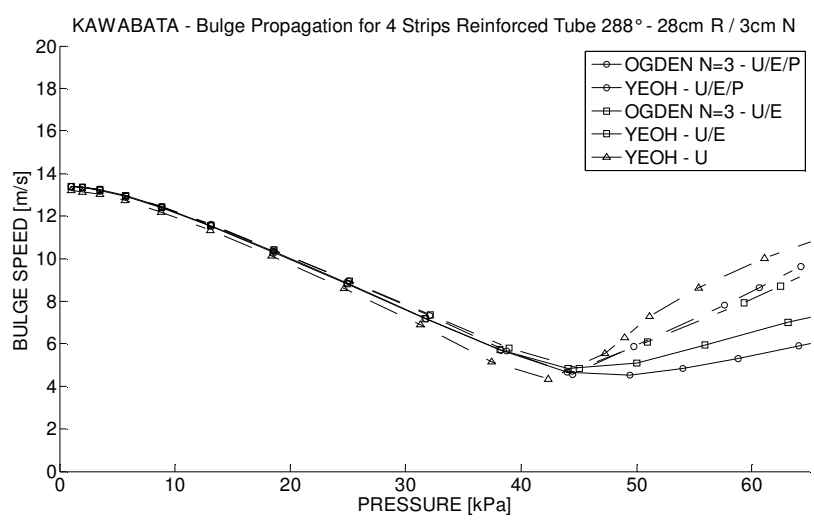

Fig. 5b Bulge wave speed sensitivity of Kawabata rubber to selected experimental data sets used 


\subsubsection{Influence of reinforcement characteristics on bulge wave speed}

To demonstrate that the existence of the reinforcement material, rather than its thickness, is critical Figure 6 provides $c_{b}-p$ plots for a 4 strips of reinforcement with coverage angles of $216^{\circ}$ and $288^{\circ}$. In each case Treloar rubber with a wall thickness of $28 \mathrm{~cm}$ and reinforcement thicknesses of $1 \mathrm{~cm}, 3 \mathrm{~cm}$ and $5 \mathrm{~cm}$ is considered.

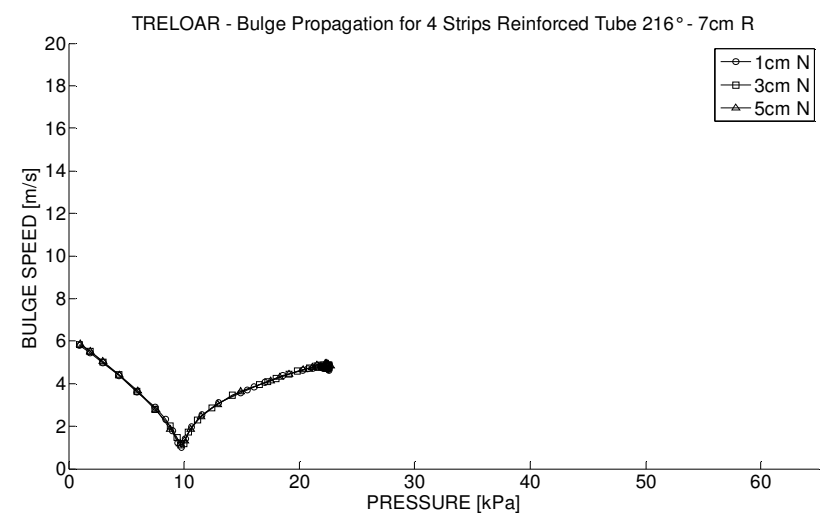

Fig. 6a Bulge wave speed versus inflation pressure for tube coverage $216^{\circ}$ and $7 \mathrm{~cm}$ rubber thickness

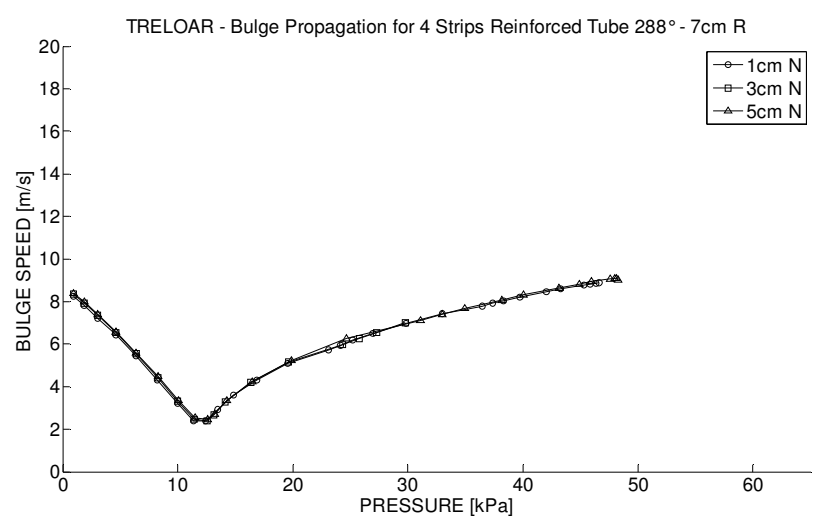

Fig. 6b Bulge wave speed versus inflation pressure for tube coverage $288^{\circ}$ and $7 \mathrm{~cm}$ rubber thickness

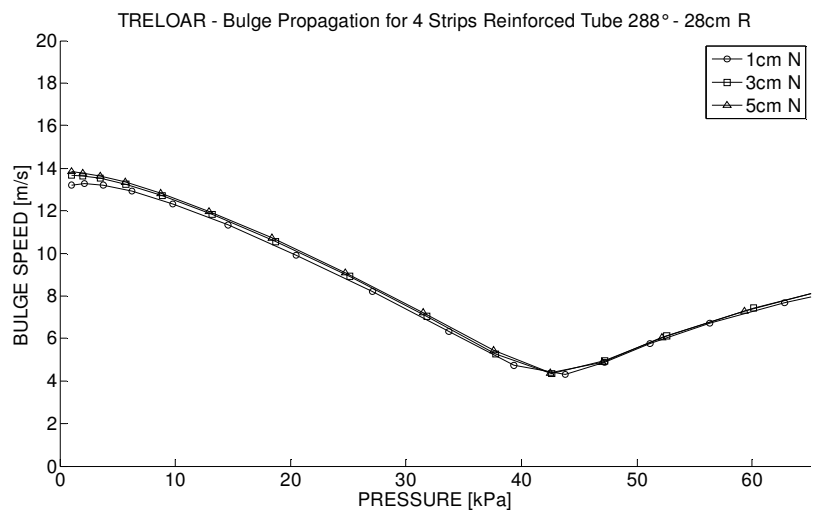

Fig. 6d Bulge wave speed versus inflation pressure for tube coverage $288^{\circ}$ and $28 \mathrm{~cm}$ rubber thickness

Figure 6 demonstrates that increasing bulge wave speed requires an increase in either tube wall thickness or angle of coverage of reinforcement material or both dependent upon frequency of external incident waves. Furthermore this conclusion is independent of the selected reinforcement thickness. 
Having considered variation in reinforcement thickness we next explore the impact of varying the Young's modulus of the Nylon-based reinforcement material. The Nylon is generally designated Nylon n-m, where $\mathrm{n} \& \mathrm{~m}$ relate to the chemical structure, and the Nylons may or may not contain fibres of reinforcement. Using reference [63] different forms of Nylon with and without reinforcement have been searched to identify a representative range of possible Young modulus values, e.g. glass reinforced Nylon 46 has values between 2.8-20 GPa. This range of Young modulus is effectively covered using multiples of 1/3, 3 and 6 of the original assumed $3 \mathrm{GPa}$ value. This range of values readily represents many different forms of Nylon. Reinforcement thickness again reverts to its original value of $3 \mathrm{~cm}$ and only 4 reinforcement strips are considered for coverage angles of $216^{\circ} \& 288^{\circ}$ and rubber wall thicknesses of $7 \mathrm{~cm} \& 28 \mathrm{~cm}$. Figures $7 \mathrm{a}$ to $\mathrm{d}$ provide variation of bulge wave speed with pressure for the described reinforcement changes.

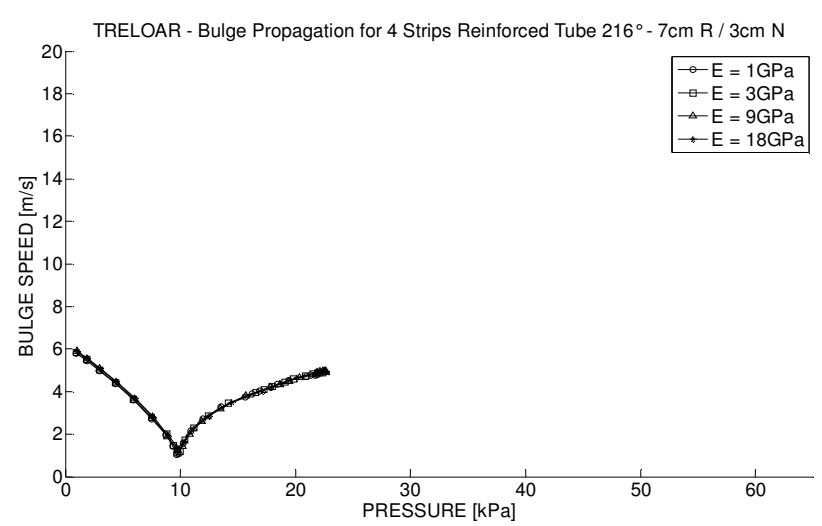

Fig. 7a Effect of $216^{\circ}$ coverage of reinforced material on bulge wave speed for a $7 \mathrm{~cm}$ Treloar rubber with different reinforcement Young modulus

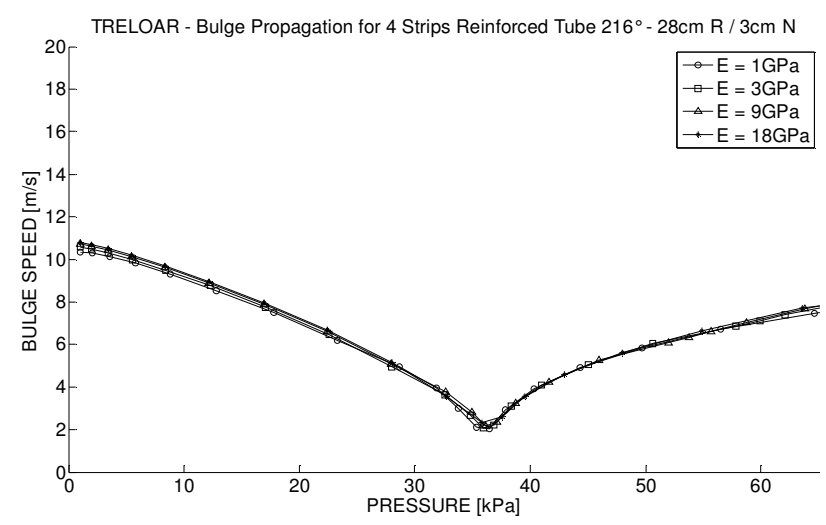

Fig. 7c Effect of $216^{\circ}$ coverage of reinforced material on bulge wave speed for a $28 \mathrm{~cm}$ Treloar rubber with different reinforcement Young modulus

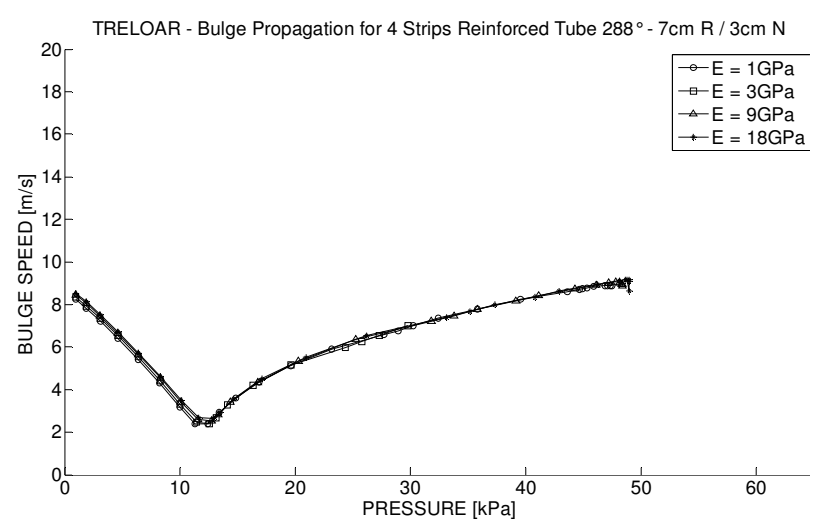

Fig. $7 \mathrm{~b}$ Effect of $288^{\circ}$ coverage of reinforced material on bulge wave speed for a $7 \mathrm{~cm}$ Treloar rubber with different reinforcement Young modulus

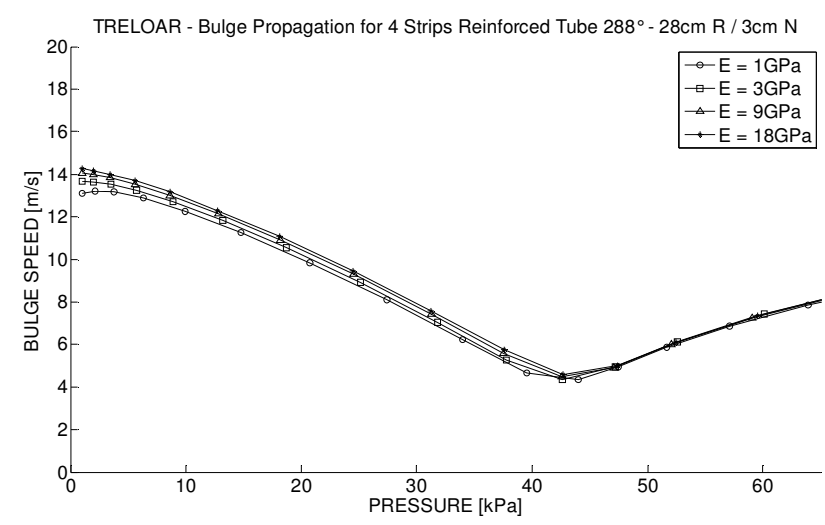

Fig. 7d Effect of $288^{\circ}$ coverage of reinforced material on bulge wave speed for a $28 \mathrm{~cm}$ Treloar rubber with different reinforcement Young modulus 
Each plot of Figure 7 indicates that the largest difference in bulge wave speed is encountered for a very small inflation pressure. Figure $7 \mathrm{~d}$ with a rubber wall thickness of $28 \mathrm{~cm}$ has the largest spread of bulge wave velocities values, indicating a variation of $8.84 \%$ as the Young's modulus is reduced over the range $1 \mathrm{GPa}$ to $18 \mathrm{GPa}$. This spread is essentially halved when the reinforcement coverage angle is reduced to $216^{\circ}$ and this is again halved when maintaining the $216^{\circ}$ angle and wall thickness is reduced to $7 \mathrm{~cm}$. As inflation pressure increases the influence of Young modulus for the reinforcement material becomes negligible.

\subsection{Device structural integrity}

The von Mises stresses presented are calculated using Equation (19b). Prior to discussing the pressure distribution over the distensible tube it is useful to recall that Equation (10) relates the hoop stress to tube radius, wall thickness and pressure. The corresponding longitudinal stress requires a factor of one-half. Since the tube ends are fully clamped the largest radial changes will take place centrally.

To compare predicted stress levels in the tube for different coverage angles (up to $288^{\circ}$ ), irrespective of wall thickness, the value of the radial stretch parameter in Figure 2 should not exceed 1.8. If we want to compare behaviour at comparable inflation pressures the workable ranges are approximately $8-15 \mathrm{kPa}, 15-25 \mathrm{kPa}$ and $30-50 \mathrm{kPa}$ for wall thicknesses of $7 \mathrm{~cm}, 14 \mathrm{~cm}$ and $28 \mathrm{~cm}$ respectively. Furthermore, since the tensile yielding stress is unknown we have selected a nominal threshold of 10MPa. Consequently, if the predicted stress exceeds this threshold the stress level, irrespective of actual value, will be shaded grey.

The $p-\lambda_{1}$ curve corresponding to the Ogden curve of Figure $3 \mathrm{a}$ is presented in Figure 8 together with stress level plots for two distinct values of radial stretch, namely $\lambda_{1}=1.94$ and $\lambda_{1}=3.48$. When the finite element analysis is undertaken for different scenarios there is no external control of the increments in either inflation pressure or radial stretch parameter. The stress distribution is recoverable for each inflation pressure applied and to provide comparative results one must seek appropriate solutions within the ensemble of solutions subject to either approximate equivalent pressure or approximate equivalent stretch parameter. 


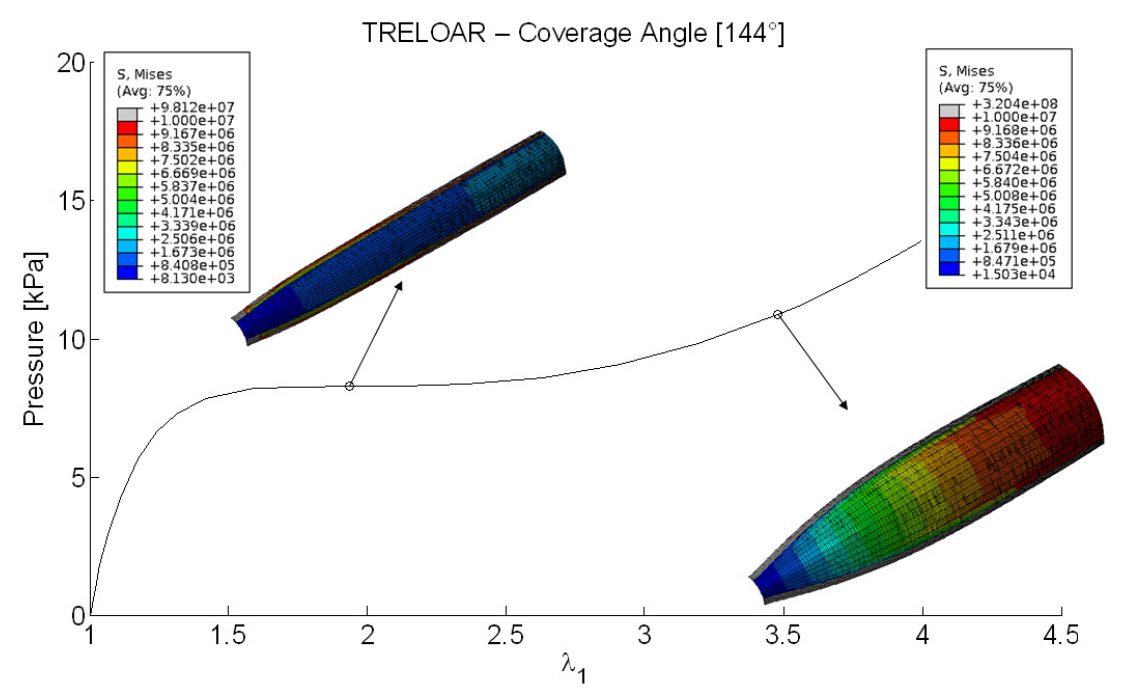

Fig. 8 Stress variation for $\left(\lambda_{1}, p\right)$ coordinates $(1.94,8.30) \&(3.48,10.86)$

Figure 8 for a coverage angle of $144^{\circ}$ clearly indicates that whilst the inflation pressures are respectively $8.30 \mathrm{kPa}$ and $10.86 \mathrm{kPa}$ the maximum stress in the first case is less than $2.5 \mathrm{MPa}$, whereas in the second case the stress is very close to the assigned (arbitrary) threshold of 10MPa. It should be noted that stress levels are lower at the clamped (left-hand) end of the tube and thereafter increase along the tube so that the highest stresses occur at the tube centre (right-hand end). In subsequent figures it is the stresses over this portion of the tube that is displayed.

In Figure 9 the nearest equivalent pressures are selected for a coverage angle of $144^{\circ}$ and a $7 \mathrm{~cm}$ wall thickness to display stress variation for different numbers of reinforcement strips. In this and subsequent figures A, B \& C respectively indicate 4,8 \& 16 reinforcement strips.

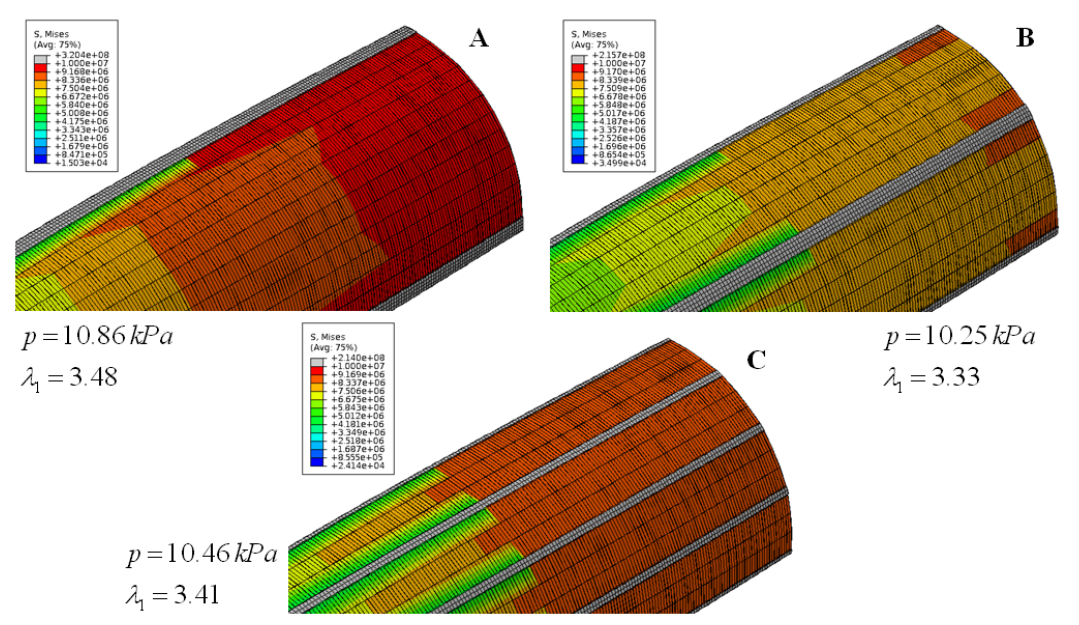

Fig. 9 Stress distribution in 4 (A), 8 (B) and 16 (C) strips for a coverage angle of $144^{\circ}$. 
Figure $3 \mathrm{a}$ indicates that the pressures associated with Figure 9 coincide with the $p-\lambda_{1}$ variations for which selection of the strain-energy function is not relevant. The grey strips in Figure 9 indicate reinforcement material, not exceedence of assigned stress threshold in the rubber. Case B may appear different to cases A \& C, which clearly have comparable high stresses, but it has the lowest pressure and the lowest stretch ratio. The extent of the high stress levels in $\mathrm{C}$ is increased in $\mathrm{A}$ as a consequence of the pressure increase being twice that between B \& $\mathrm{C}$ for an equal variation of stretch parameter. More importantly one notices that the uniformly high stress of A is essentially repeated between the reinforcement strips of $\mathrm{C}$. This seems to suggest that the stress levels experienced is not significantly influenced by number of reinforcement strips.

In Figure 9 the pressure and the stretch parameter values were all bounded within a small interval. In Figure $10 \mathrm{a} \& \mathrm{~b}$ the coverage angle is $288^{\circ}$ and again we consider $4,8 \& 16$ strips. In Figure 10a the finite element discretisation is the same as that employed in Figure 9, but we have selected the stress distribution on the basis of comparable radial stretch ratios. In the case of 16 strips the rubber material is represented by one element between the reinforcement strips. This approach may seem too crude for modelling the pure rubber portions of the tube. Therefore, Figure $10 \mathrm{~b}$ provides a corresponding analysis for radial stretch ratios of the same order of magnitude with the number of circumferential and longitudinal elements doubled. Further element refinement may be achieved by tripling the number of circumferential and longitudinal elements and continuing to maintain an element aspect ratio of unity. This approach means that the inextensible reinforcement material has unwanted refinement. As the number of strips increases so the number of circumferential elements between strips edges reduces. Therefore a fourth mesh has been developed whereby the number of elements modelling the inextensible material is reduced to permit greater refinement of the unstiffened rubber. However the total number of elements remains the same of that of mesh 3 . Figure $10 \mathrm{c}$ then provides the stress variation for 16 strips based on meshes $3 \& 4$. For completeness variation of the four meshes is captured in Figure 10d, whereas both mesh influence and variation of the number of strips upon $p-\lambda_{1}$ changes is captured in Figure 10e, a redevelopment of Figure $3 c$. 


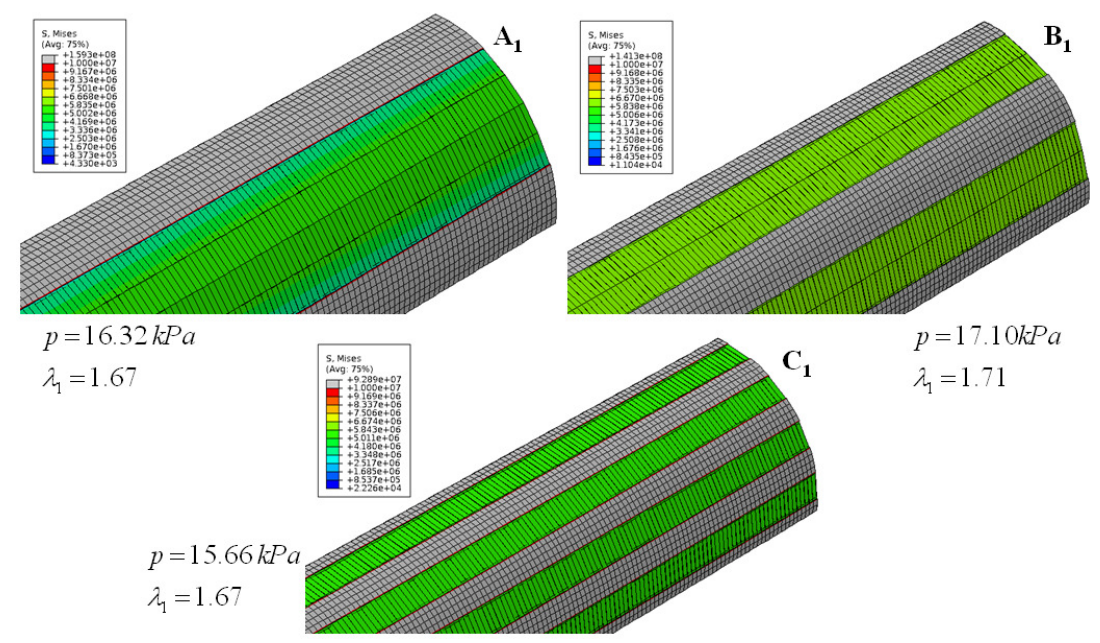

Fig. 10a Stress distribution in $4\left(A_{1}\right), 8\left(B_{1}\right)$ and $16\left(C_{1}\right)$ strips for a coverage angle of $288^{\circ}$

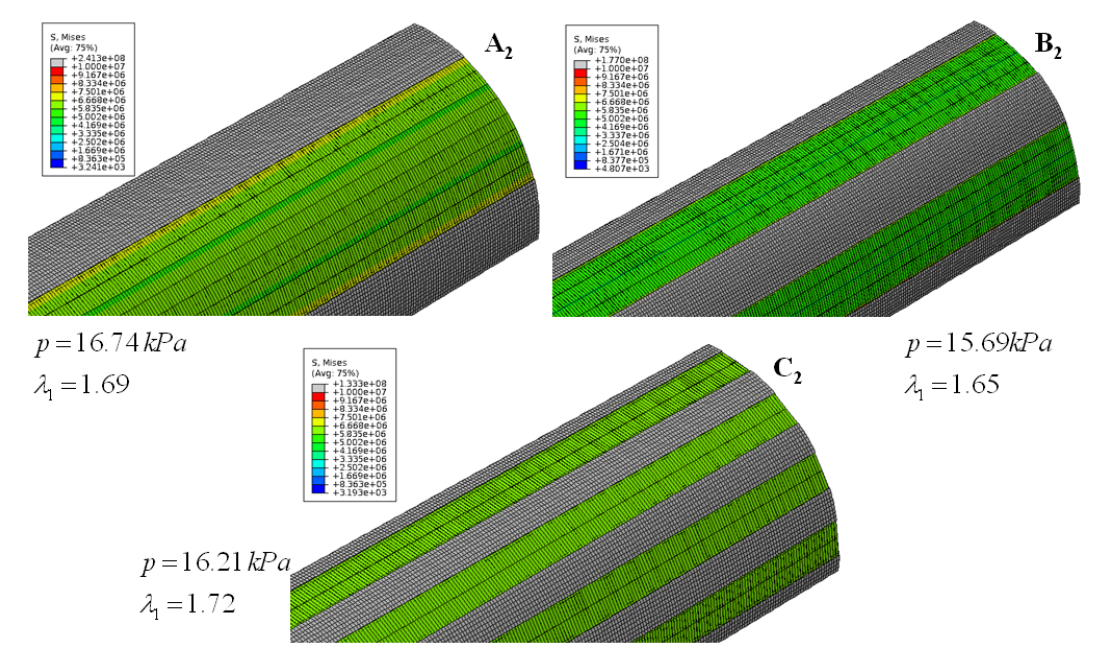

Fig. 10b Stress distribution in $4\left(\mathrm{~A}_{2}\right), 8\left(\mathrm{~B}_{2}\right)$ and $16\left(\mathrm{C}_{2}\right)$ strips for a coverage angle of $288^{\circ}$ using quadruple number of rubber and reinforcement elements

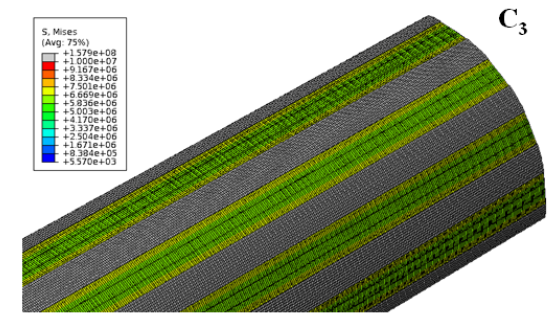

$p=15.86 \mathrm{kPa}$

$\lambda_{1}=1.72$

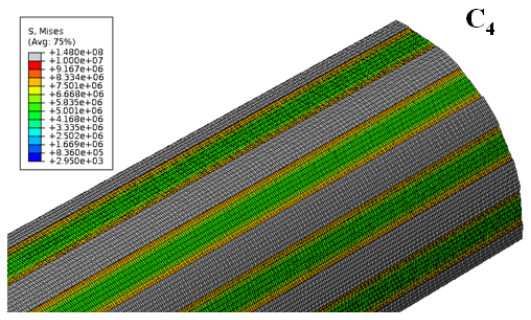

$p=15.84 \mathrm{kPa}$

$\lambda_{1}=1.71$

Fig. 10c Stress distribution in 16 strips using further finite element refinements:

$\mathrm{C}_{3}$ is a tripling of circumferential and longitudinal element

$\mathrm{C}_{4}$ has reduced modelling of stiffened portions to facilitate further refinement of pure rubber portion 

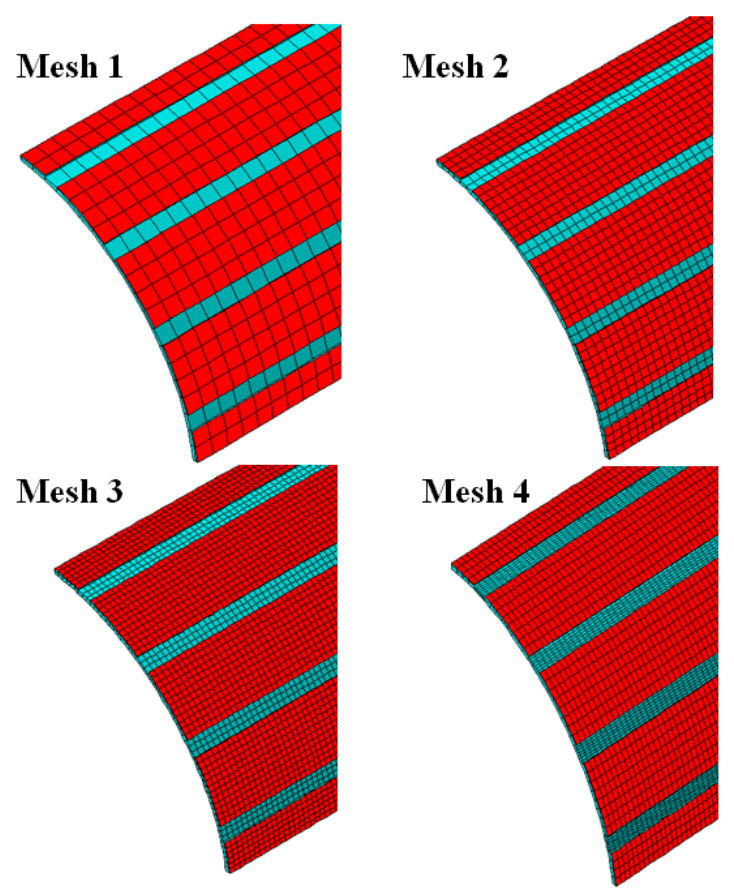

Fig. 10d Further mesh refinement for the 16 strips of reinforcement material

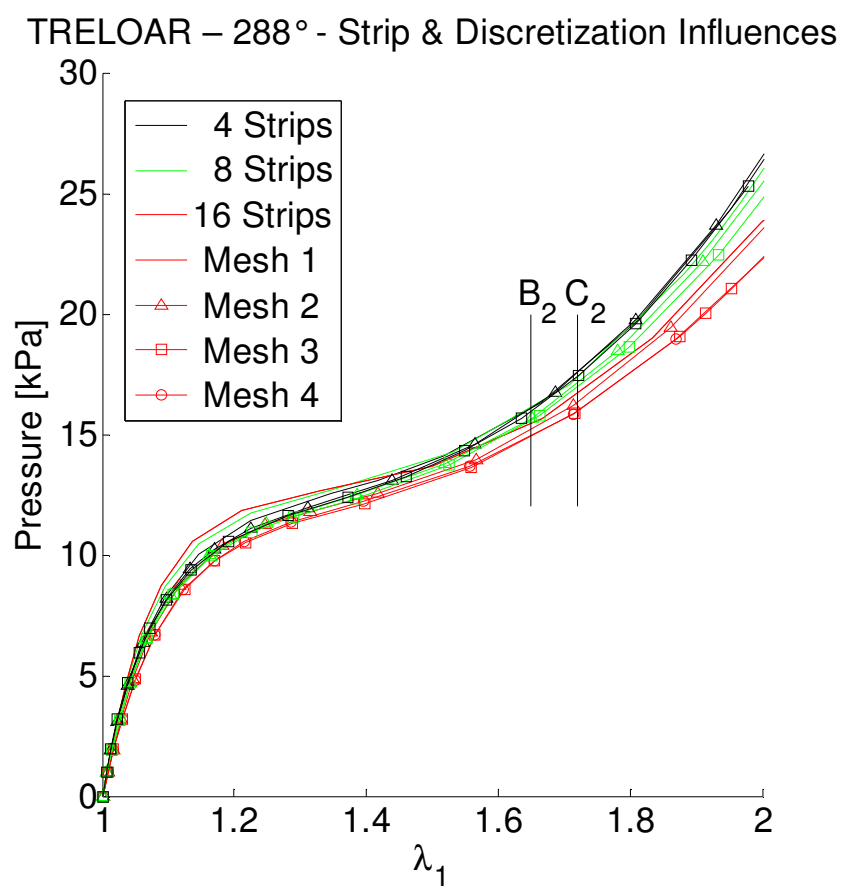

Fig. 10e Repeated analysis of Figure 4c using refined mesh of Figure 11d

Figure 10 clearly demonstrate that the initial finite element discretisation is sufficient to model the reinforced rubber tube irrespective of the number of reinforcement strips considered. Having essentially maintained the same nominal value of radial stretch parameter in Figure 10a to c, we note that the inflation pressure associated with each $\lambda_{1}$ value lies within an expected interval. Quadrupling the number of rubber and reinforcement elements leads to insignificant differences in stress levels when comparing Figure 10a with Figure 10b. Figure 10c represents a further 
refinement which more than double the number of elements involved in Figure 10b. However the stress levels remain essentially the same with little variation in the associated pressure for the same $\lambda_{1}$ values. Figures $10 \mathrm{~d} \&$ e permit the reader to appreciate the discretisation refinement undertaken and the almost insignificant impact the number of strips and the mesh variations have on the $p-\lambda_{1}$ plots.

The influence of rubber thickness is captured in Figure 11 for a reinforcement coverage angle of $144^{\circ}$ and 4 reinforcement strips.

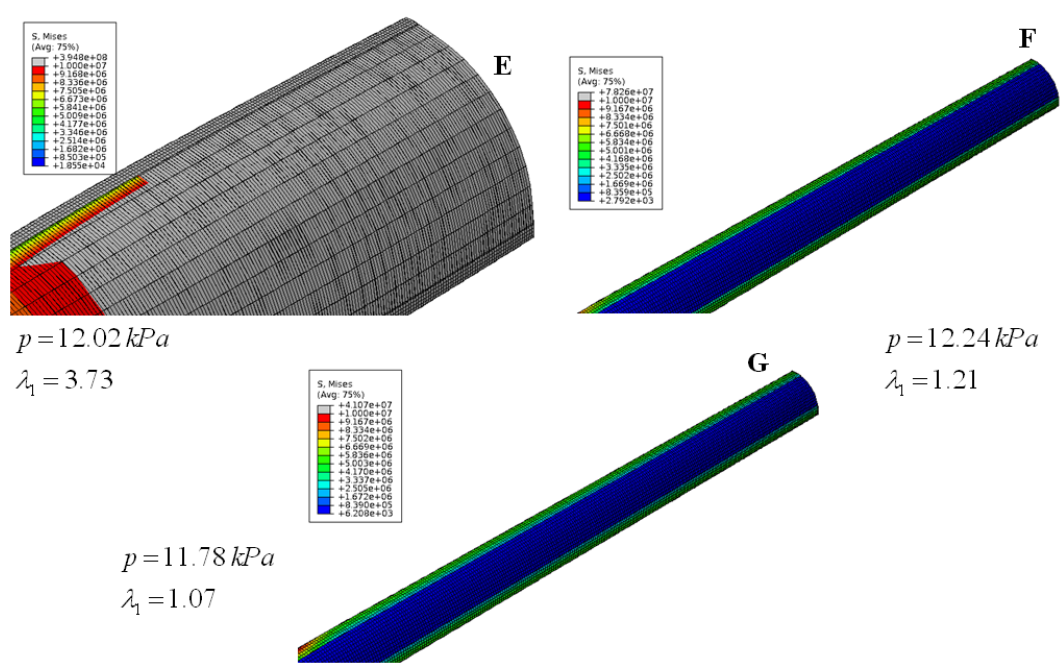

Fig. 11a Variation of stress distribution for wall thicknesses of $7 \mathrm{~cm}(\mathrm{E}), 14 \mathrm{~cm}(\mathrm{~F})$ and $28 \mathrm{~cm}(\mathrm{G})$, for an inflation pressure of approximately $12 \mathrm{kPa}$ with 4 strips of reinforcement and a coverage angle of $144^{\circ}$.

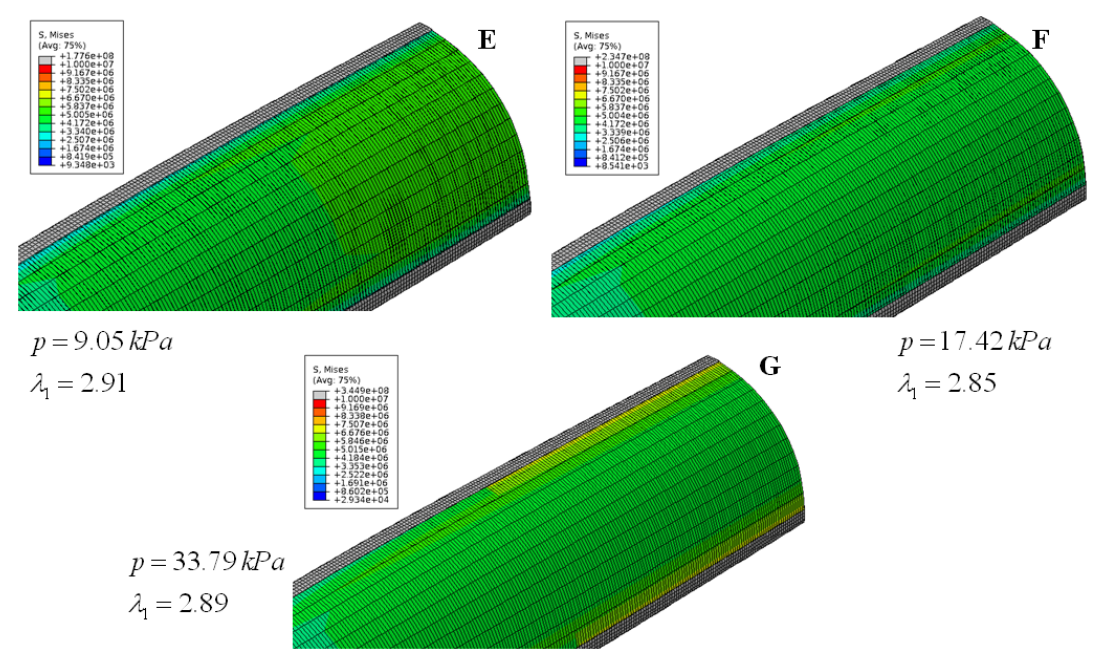

Fig. 11b Variation of stress distribution for wall thicknesses of $7 \mathrm{~cm}(\mathrm{E}), 14 \mathrm{~cm}(\mathrm{~F})$ and $28 \mathrm{~cm}(\mathrm{G})$, for a radial stretch ratio of approximately 2.9 , with 4 strips of reinforcement and a coverage angle of $144^{\circ}$. 
In Figure 11a an inflation pressure of $12 \mathrm{kPa}$ is the pressure above the hydrostatic within and outside the tube. It is clear that as the thickness increases Figure 11a (E to $G$ ) so the radial stretch ratio, required to sustain the inflation pressure, is reduced. Thus for the $7 \mathrm{~cm}$ wall thickness the stresses have exceeded the assigned threshold entirely in the central region of the tube, upon doubling the thickness the radial increase is less than a third of the previous case and the stress levels are well below 10MPa. G has again very low stresses because the inflation pressure achieves less of $7 \%$ of inflated radius. Figure $11 \mathrm{~b}$ indicates that, for essentially the same nominal increase in radius, the stresses are comparable although the inflation pressure required to achieve the increased radius is essentially doubled as wall thickness is doubled.

\section{Conclusion and final comments}

The analysis reported indicates that avoidance of aneurysm initiation and matching of internal bulge wave speed to external incident wave speed may be accomplished through a judicious selection of distensible tube thickness and a sufficiently large outer coverage of the tube with an inextensible material. The inextensible reinforcement material has a Young's modulus significantly greater than the corresponding 'equivalent' Young's modulus of the tube. Hence expected variations associated with different nylon based materials are of little consequence on the overall performance of the device. Similarly the thickness of the reinforcement strips has little impact on bulge wave speed.

The number of strips to avoid aneurysm initiation does not appear to be a design constraint. The pressure, the bulge wave speeds and the peak stress levels are essentially independent of the number of constituent strips.

The application of the noted modified von Mises criterion requires more detailed information regarding the compressive and tensile yield stresses of the selected tube material. This in term would permit a more appropriate selection of stress threshold once a particular rubber has been selected.

The tube thickness for the assumed tube length and diameter is clearly going to provide construction challenges assuming that economic viability is demonstrable. Current joint investigation by TARRC and the University of Southampton are addressing the long term behaviour of different rubbers to cyclic loading and their reactions to exposure to ozone et cetera. 


\section{Acknowledgments}

Reported research was partially funded through the EPSRC grant (EP/F030975/1) addressing the hydrodynamics of a distensible wave energy converter. Additional funding was made available through University of Southampton internal funds.

\section{References}

[1] S.H. Salter, Wave Power, Nature 249 (1974) 720-724.

[2] S.H. Salter, D.C. Jeffrey, J.R.M. Taylor, The architecture of nodding duck wave power generators, The Naval Architect, RINA January (1976) 21-24.

[3] M. Katory, Application of theoretical hydrodynamics to the design of wave power generators, The Naval Architect, RINA May (1976) 91-92.

[4] M. Katory, On the motion analysis of large unsymmetric bodies among sea waves, The Naval Architect, RINA September (1976) 158-159.

[5] G.E. Hearn, E. Donati, I.K. Mahendran, Prediction, Measurement and Comparison of FluidStructure Interaction using Mathematical and Experimental Results, Applied Mathematical Modelling, 7 (1983) 41-47

[6] L.J Ducker, Reinforced Membrane for the Clam Wave Energy Device, Proceedings of $9^{\text {th }}$ International Offshore \& Polar Conference, Brest, France, May-June (1999), 174-179.

[7] M. Katory, On the motion analysis of interlinked articulated bodies floating among sea waves, The Naval Architect, RINA January (1977) 28-29.

[8] G.E. Hearn, M. Katory, Application of Hydrodynamics Analysis to Wave Power Generators, Journal of Institute of Fuel, 119 (1978) 119-126.

[9] J.N. Newman, Absorption of wave energy by elongating bodies, Applied Ocean Research, 1 (1979) 189-196.

[10] R. Yemm, D. Pizer, C. Reztler, R. Henderson, Pelamis: experience from concept to connection Philosophical Transactions of the Royal Society of London - Series A, 370 (2012) 365-380 [11] A.W. Stahl, The utilization of the power of the oceans, Transactions of American Society of Mechanical Engineers, 13 (1892) 438-506.

[12] R.G. Standing, Use of Potential Theory in Evaluating Wave Forces on Offshore Structures, in:

B. Count (Ed.), Proceedings of the IMA International Conference on Power from Sea Waves, Academic Press, 1980, pp. 175-212. 
[13] D.V. Evans, A theory for wave-power absorption by oscillating bodies, Journal of Fluid Mechanics, 77 (1976) 1-25.

[14] M.J. Lighthill, Two dimensional analyses related to wave energy extraction by submerged resonant ducts, Journal of Fluid Mechanics, 91 (1979) 253-318.

[15] B.M. Count, On the dynamics of wave power devices, Proceedings of the Royal Society of London - Series A, 363 (1978) 559-579.

[16] C.C. Mei, Power extraction from water waves, Journal of Ship Research, 20 (1976) 63-66.

[17] G.E. Hearn, J.R. Chaplin, The Fluid Structure Interaction of Wave Energy Devices: Some Old and Some New Theoretical and Experimental Challenges, International Wave Energy Conference, organised by Royal Institution of Naval Architects (RINA), London (2010).

[18] M. Katory, On the hydrodynamic design of pneumatic type wave power generators, The Naval Architect, RINA May (1977) 100-101.

[19] T.V. Heath, A review of oscillating water columns, Philosophical Transactions of Royal Society of London - Series A, 370 (2012) 235-245.

[20] M. Takao, T. Setoguchi, Air Turbines for Wave Energy Conversion, International Journal of Rotating Machinery (2012), Article ID 717398, 10pp.

[21] G.E. Hearn, D. Lafforgue, E. Perdriset, D. Saydan, The Hydrodynamic and Dynamic Motion Analysis of a Damaged Ship, The International Journal of Maritime Engineering Transactions RINA Part A, June (2008) 13-36.

[22] J. Falnes, K. Budal, Wave power conversion by point absorbers, Norwegian Maritime Research, 6 (1978) 2-11.

[23] J. Falnes, J. Hals, Heaving buoys, point absorbers and arrays, Philosophical Transactions of Royal Society of London - Series A, 370 (2012) 246-277.

[24] T.S. Parel, M.D. Rotaru, J.K. Sykulski, G.E. Hearn, Optimisation of a tubular linear machine with permanent magnets for wave energy extraction, COMPEL, The International Journal for Computation and Mathematics in Electrical and Electronic Engineering, 30 (2011) 1056-1068. [25] R. Antonutti, G.E. Hearn, Optimisation of Point-Absorber Arrays: Proceedings of the $9^{\text {th }}$ European Wave and Tidal Energy Conference (EWTEC), Southampton (2011), September. [26] M.A. Mueller, Electrical generators for direct drive wave energy conversion, IEE Proceedings of Generation, Transmission and Distribution, 149 (2002) 446-456.

[27] M. Leijon, C. Boström, O. Danielsson, S. Gustafsson, K. Haikonen, O. Langhamer, E. Strömstedt, M. Stålberg, J. Sundberg, O. Svensson, S. Tyrberg, R. Waters, Wave Energy from the North Sea: Experiences from the Lysekil Research Site, Surveys in Geophysics, 29 (2008) 221-224 
[28] F. Kara, Time domain prediction of power absorption from ocean waves with latching control, Renewable Energy, 35 (2010) 423-434.

[29] F.J.M. Farley, R.C.T. Rainey, Radical Design Options for Wave-Profiling Wave Energy Converters, $21^{\text {st }}$ International Workshop on Water Waves and Floating Bodies, Loughborough (2006), April 2-5.

[30] J.R. Chaplin, F.J.M. Farley, M.E. Prentice, R.C.T. Rainey, S.J. Rimmer, A.T. Roach, Development of the Anaconda All-Rubber WEC, $7^{\text {th }}$ European Wave and Tidal Conference, Lisbon (2007), September, 1-13.

[31] J.R. Chaplin, V. Heller, F.J.M. Farley, G.E. Hearn, R.C.T. Rainey, Laboratory testing the Anaconda, Philosophical Transaction of the Royal Society of London. Series A, 370 (2012) 403404.

[32] M.J. Lighthill, Waves in fluids, Cambridge University Press, Cambridge, 1978.

[33] F.J.M. Farley, R.C.T. Rainey, J.R. Chaplin, Rubber tubes in the sea, Philosophical Transactions of the Royal Society of London - Series A, 370 (2012) 381-402.

[34] J. Crighton Bramwell, A.V. Hill, The Velocity of the Pulse Wave in Man, Proceedings of the Royal Society of London - Series B, Containing Papers of a Biological Character, 93 (1922) 298306.

[35] J.J. Stoker, Water Waves - The Mathematical Theory with Applications, Interscience Publishers Inc., New York, 1957.

[36] J.M. Gere, B.J. Goodno, Mechanics of Materials, 7th edition, Cengage Learning, Stamford, 2008.

[37] A. Mallock, Note on the Instability of India-Rubber Tubes and Balloons when Distended by Fluid Pressure, Proceedings of the Royal Society of London, 49 (1890-1891) 458-463.

[38] S. Kyriakides, Y.C. Chang, On the inflation of a long elastic tube in the presence of axial load, International Journal of Solids and Structures, 26 (1990) 975-991.

[39] S. Kyriakides, Y.C. Chang, The initiation and propagation of a localized instability in an inflated elastic tube, International Journal of Solids and Structures, 27 (1991) 1085-1111.

[40] J. Shi, G.F. Moita, The post-critical analysis of axisymmetric hyper-elastic membranes by the finite element method, Computer Methods in Applied Mechanics and Engineering, 135 (1996) 265281.

[41] A. Needleman, Inflation of spherical rubber balloons, International Journal of Solids and Structures, 13 (1977) 409-421

[42] D.M. Haughton, Post-bifurcation of Perfect and Imperfect Spherical Elastic Membranes, International Journal of Solids and Structures, 16 (1980) 1123-1133. 
[43] E. Chater, J.W. Hutchinson, On the propagation of bulges and buckles, Journal of Applied Mechanics, 51 (1984) 269-277.

[44] X. Guo, Kinematic modeling of finite axisymmetric inflation for an arbitrary polymeric membrane of revolution, Polymer-Plastics Technology and Engineering, 40 (3) (2001) 341-361. [45] X. Guo, Large deformation analysis for a cylindrical hyperelastic membrane of rubber-like material under internal pressure, Rubber Chemistry and Technology, 74 (2001) 100-115.

[46] D. Tang, C. Yang, D.N. Ku, A 3-D thin-wall model with fluid-structure interactions for blood flow in carotid arteries with symmetric and asymmetric stenoses, Computers and Structures, 72 (1999) 357-377.

[47] M. Kohandel, S. Sivaloganathan, G. Tenti, J.M. Drake, The constitutive properties of the brain parenchyma Part 1. Strain energy approach, Medical Engineering \& Physics, 28 (2006) 449-454. [48] Z. Gao, K. Lister, J.P. Desai, Constitutive Modeling of Liver Tissue: Experiment and Theory, Annals of Biomedical Engineering, 38 (2010) 505-530.

[49] R.W. Ogden, Large deformation isotropic elasticity - on the correlation of theory and experiment for incompressible rubber-like solids, Proceedings of the Royal Society of London, 326 (1972) 565-584.

[50] A. Bucchi, G.E. Hearn, Predictions of Aneurysm Formation in Distensible Tubes - Part A Theoretical Background to Alternative Approaches, International Journal of Mechanical Sciences, XX (2012) YYY-YYY.

[51] A. Bucchi, G.E. Hearn, Predictions of Aneurysm Formation in Distensible Tubes - Part B Application and Comparison of Alternative Approaches, International Journal of Mechanical Sciences, XX (2012) YYY-YYY.

[52] O.H. Yeoh, Some forms of the strain-energy function for rubber, Rubber Chemistry and Technology, 66 (1993) 754-771.

[53] E.H. Twizell, R.W. Ogden, Non-linear optimization of the material constants in Ogden's stressdeformation function for incompressible isotropic elastic materials, Journal of Australian Mathematical Society, Series B, 24 (1983) 424-434.

[54] S.P. Timoshenko, J.N. Goodier, Theory of Elasticity, 3rd edition, McGraw Hill Kogakusha, Japan, 1970.

[55] L.R. Wang, Z.H. Lu, I. Hagiwara, Finite element simulation of the static characteristics of a vehicle rubber mount, Proceedings of the Institution of Mechanical Engineers, Part D: Journal of Automobile Engineering, 216 (2002) 965-973.

[56] L.R.G. Treloar, Stress-strain data for vulcanised rubber under various types of deformation, Transactions of the Faraday Society, 40 (1944) 59-70. 
[57] S. Kawabata, M. Matsuda, K. Tei, H. Kawai, Experimental Survey of the Strain Energy Density Function of Isoprene Rubber Vulcanizate, Macromolecules, 14 (1981) 154-162.

[58] E. Riks, An Incremental Approach to the Solution of Snapping and Buckling Problems, International Journal of Solids and Structures, 15 (1979) 529-551.

[59] T. Sussman, K.J. Bathe, A finite element formulation for nonlinear incompressible elastic and inelastic analysis, Computers \& Structures, 26 (1987) 357-409.

[60] R.S. Rivlin, D.W. Saunders, Large Elastic Deformations of Isotropic Materials. VII.

Experiments on the Deformation of Rubber, Philosophical Transactions of Royal Society, 243

(1951) 251-288.

[61] A.G. James, A. Green, G.M. Simpson, Strain Energy Functions of Rubber. I. Characterization of Gum Vulcanizates, Journal of Applied Polymer Science, 19 (1975) 2033-2058.

[62] A.G. James, A. Green, Strain Energy Functions of Rubber. II. The Characterization of Filled Vulcanizates, Journal of Applied Polymer Science, 19 (1975) 2319-2330.

[63] MatWeb Material Property Data accessed via www.matweb.com (2012). Thereafter one clicks possible materials of interest to acquire complete data sets. 